\title{
Nanoscopic dopamine transporter distribution and conformation are inversely regulated by excitatory drive and $D_{2}$-autoreceptor activity
}

\author{
Matthew D. Lycas ${ }^{1}$, Aske L. Ejdrup ${ }^{1}$, Andreas T. Sørensen ${ }^{1}$, Nicolai O. Haahr ${ }^{1}$, Søren H. Jørgensen ${ }^{1}$, Daryl \\ A. Guthrie ${ }^{2}$, Jonatan F. Støier ${ }^{1}$, Christian Werner ${ }^{3}$, Amy Hauck Newman², Markus Sauer $^{3}$, \\ Freja Herborg ${ }^{1}$ and Ulrik Gether ${ }^{1,4, *}$
}

\begin{abstract}
${ }^{1}$ Molecular Neuropharmacology and Genetics Laboratory, Department of Neuroscience, Faculty of Health and Medical Sciences, Maersk Tower 7.5, University of Copenhagen, DK-2200 Copenhagen, Denmark
\end{abstract}

\begin{abstract}
${ }^{2}$ Medicinal Chemistry Section, Molecular Targets and Medications Discovery Branch, National Institute on Drug Abuse-Intramural Research Program, National Institutes of Health, Baltimore, Maryland 21224

${ }^{3}$ Department of Biotechnology and Biophysics, Biocenter, Julius-Maximilians-Universität Würzburg, Am Hubland, 97074 Würzburg, Germany
\end{abstract}

\footnotetext{
${ }^{4}$ Lead contact

*Correspondence: gether@sund.ku.dk
} 


\section{SUMMARY}

The nanoscopic organization and regulation of individual molecular components in presynaptic varicosities of neurons releasing modulatory volume neurotransmitters like dopamine (DA) remain largely elusive. Here we show by application of several single-molecule sensitive super-resolution microscopy techniques to cultured neurons and mouse striatal slices, that the dopamine transporter (DAT), a key protein in varicosities of dopaminergic neurons, exists in the membrane in dynamic equilibrium between an inward-facing nanodomain-localized and outward-facing unclustered configuration. The balance between these configurations is inversely regulated by excitatory drive and by DA D2-autoreceptor activation in manner dependent on $\mathrm{Ca}^{2+}$-influx via $\mathrm{N}$-type voltage-gated $\mathrm{Ca}^{2+}$-channels. The DAT nanodomains contain tens of transporters molecules and overlap with nanodomains of PIP2 (phosphatidylinositol-4,5-bisphosphate) but show little overlap with D2-autoreceptor, syntaxin-1 and clathrin nanodomains. By demonstrating that nanoscopic reorganizations with putative major impact on transmitter homeostasis can take place in dopaminergic varicosities, the data have important implications for understanding modulatory neurotransmitter physiology.

Keywords: super-resolution microscopy; dSTORM; PALM, expansion microscopy; neurotransmitter transporters; dopamine transporter; dopamine receptors; PIP2; fluorescent cocaine analogues; volume transmission; synaptic architecture; nanodomains 


\section{INTRODUCTION}

The emergence of super-resolution imaging techniques provides unprecedented possibilities for improving our insights into the molecular organization and dynamic regulation of pre- and postsynaptic structures in the brain. Indeed, it has become clear that regulatory processes of fundamental importance for spatial and temporal control of synaptic signaling occur at a nanoscale level and that these processes can neither be visualized nor quantitatively analyzed with classical microcopy (Choquet and Hosy, 2020; Groc and Choquet, 2020; Liu and Kaeser, 2019). In excitatory synapses, it is now believed that e.g. AMPAtype and NMDA-type ionotropic glutamate receptors segregate into distinct nanodomains in the postsynaptic density and that this co-organization plays a critical role in synaptic physiology (Choquet and Hosy, 2020; Groc and Choquet, 2020).

Much less is known about the nanoscale architecture of the presynaptic compartment in neurons releasing modulatory transmitters like dopamine (DA). DA neurons project from the midbrain to the basal ganglia, the limbic system and the prefrontal cortex to mediate the role of DA in motor functions, reward mechanisms and learning processes (Bjorklund and Dunnett, 2007; Iversen, 1975; Tritsch and Sabatini, 2012). The DA neurons have remarkable axonal arbors with numerous release sites (i.e. varicosities) that enable single DA neurons to affect the function of multiple target neurons (Descarries et al., 1996; Giguere et al., 2019). DA neurotransmission, however, differs from classical fast synaptic transmission by operating perhaps primarily via "volume transmission", that is, DA is predominantly released from nonsynaptic release sites to act on target cells often located microns away (Borroto-Escuela et al., 2018; Descarries et al., 1996). It remains nonetheless elusive how the different molecular components in the dopaminergic release sites need to be organized at a nanoscale level and individually regulated to enable proper neurotransmitter release and reuptake.

A key regulator of DA neurotransmission, the dopamine transporter (DAT), is highly expressed in the varicosities of DA neurons and removes released DA from the extracellular space. DAT has received 
attention as the primary target for therapeutics, such as methylphenidate, and drugs of abuse such as methamphetamine and cocaine (German et al., 2015; Kristensen et al., 2011; Torres and Amara, 2007). Not surprisingly, DAT is subject to tight regulation through several mechanisms involving posttranslational modifications, protein-protein interactions and protein-lipid interactions (Bermingham and Blakely, 2016; Eriksen et al., 2010; German et al., 2015; Kristensen et al., 2011; Ramamoorthy et al., 2011; Torres, 2006). Moreover, we recently showed by application of super-resolution imaging that DAT regulation might involve mechanisms that cannot be revealed by more classical methods (RahbekClemmensen et al., 2017). Our data showed that DAT localizes to discrete nanodomains in the plasma membrane of cultured DA neurons and that activation of NMDA-receptors reduced DAT nanodomain localization (Rahbek-Clemmensen et al., 2017). Although this indicated that nanoscale dynamics in dopamine varicosities could be critical for controlling DA neurotransmission, the mechanisms and functional implications remain unknown.

Here we use several super-resolution imaging techniques, including Photoactivated Localization Microscopy (PALM) (Betzig et al., 2006), direct Stochastic Optical Reconstruction Microscopy (dSTORM) (Heilemann et al., 2008; Huang et al., 2009), 10 x Expansion Microscopy (ExM) (Chen et al., 2015; Tillberg et al., 2016; Truckenbrodt et al., 2019) and single-particle tracking dSTORM (spt-dSTORM) (Manley et al., 2008) together with new fluorescent cocaine-analogues (Guthrie et al., 2020) and new data analysis tools, to investigate how DAT and associated molecular components at DA varicosities are dynamically regulated. In summary, our experimental efforts demonstrate that excitatory drive and D2-autoreceptor activity inversely regulate in a $\mathrm{Ca}^{2+}$ dependent manner the conformational state of DAT and its localization in DA varicosities to PIP2 enriched nanodomains separate from syntaxin-1 (STX-1), D2-autoreceptor or clathrin nanodomains. In this way, our study uncovers a hitherto unknown level of regulation of an essential membrane protein in a modulatory transmitter terminal. 


\section{RESULTS}

\section{DAT shows nanodomain distribution in cultured neurons and striatal slices}

Our previous dSTORM work showed that DAT distributes into nanodomains at DA varicosities of cultured midbrain DA neurons (Rahbek-Clemmensen et al., 2017). To qualitatively assess the distribution of DAT regardless of potential antibody-induced clustering artifacts, we magneto-transfected cultured DA neurons with DAT having the photoconvertible fluorescent protein mEOS2 (Baker et al., 2010) fused to the N-terminus. Images of tyrosine hydroxylase $(\mathrm{TH})$ positive varicosities revealed a clustered distribution of the mEOS2-DAT signal surrounding the cytosolic TH signal (Figure 1A-D), similar to that seen by imaging the endogenous DAT by dSTORM (Rahbek-Clemmensen et al., 2017). We next employed astigmatic 3DdSTORM to show that the DAT nanodomains were localized to the periphery of the varicosities, substantiating that DAT is found mainly in the plasma membrane (Figure $1 \mathrm{E}-\mathrm{H}$ ). To assess the distribution of DAT in intact tissue, we analyzed coronal mouse brain slices (Figure 1I-L). Widefield imaging exposed the DAT positive varicosities and by dSTORM we could visualize DAT in single varicosities, showing a nanodomain distribution like that seen in cultured neurons (Figure 1I-L). To exclude that the observed distribution of DAT was not caused by artifacts introduced by the single molecule localization microscopy approach (e.g., "overcounting" (Shivanandan et al., 2014)), we turned to ExM where nanoscale spatial resolution is obtained by expanding the tissue sample using a polymer system (Chen et al., 2015). We employed a 10x expansion protocol (Truckenbrodt et al., 2019) to mouse striatal slices before DAT imaging using a spinning disk confocal microscope (Figure 1M-P). In the stained expanded sample, we captured the intermingled axonal network of DA neurons while at the same time observing clustering of DAT into nanodomains in varicosities (Figure 1M-P). Summarized, the distribution of DAT into nanodomains in the plasma membrane of DA neurons was substantiated by several high-resolution imaging techniques. 
To enable quantification of the clustered distribution of DAT, we employed the DBSCAN algorithm (density based spatial clustering of applications with noise) (Ester et al., 1996; Rahbek-Clemmensen et al., 2017) to our dSTORM data (Figure 1Q-S). We decided to extract two key parameters from the analysis: the size of the nanoclusters and the density of localizations within the clusters. The size was quantified as the fraction of clusters with a diameter $>75 \mathrm{~nm}$ (Figure 1R). By assessing the density of localizations as well, we could determine changes in the number of molecules in a cluster without a change in cluster size. Dense clusters were defined as clusters with $>80$ localizations within a radius of $50 \mathrm{~nm}$ (dense clusters in red) (Figure 1S). We also employed Voronoi tessellation to compare localization density distributions in a parameter free fashion (Levet et al., 2019). In this use of Voronoi tessellation, clusters themselves were not identified but localization density changes could be detected with greater sensitivity (Figure 1T).

We also wanted to estimate how many DAT molecules are present in an average DAT nanodomain defined by the DBSCAN analysis. We used a protocol modified from (Ehmann et al., 2014; Siddig et al., 2020) and labeled cultured DA neurons with sequential dilutions of the primary DAT antibody. This enabled us to define that a minimum cluster is made from three localizations and that an average large cluster contains $\sim 33$ copies of the antibody corresponding to 50-100 DAT molecules as a rough estimate (Figure S1). The same data series lent itself perfectly for cluster analysis by varied label density cluster verification, enabling us to distinguish between random clustering arising from multiple observations of single fluorophores and true clustering (Baumgart et al., 2016). In agreement with our data in DAT expressing Cath.a-differentiated (CAD) cells (Rahbek-Clemmensen et al., 2017), our analysis supported that the clustering behavior of DAT in cultured neurons reflect true nanocluster formation (Figure S2).

\section{Nanodomain localized DAT is preferentially in an inward-facing conformation}

To assess putative functional implications of the nanoclustered distribution of DAT, we investigated whether this could involve a conformational bias with nanoclustered DAT having a different conformation 
than unclustered DAT. Cocaine and cocaine-like inhibitors are known to bind with higher affinity to DAT in the outward-facing conformation than in the inward-facing conformation (Beuming et al., 2008; Loland et al., 2008; Newman et al., 2019). We envisioned accordingly (and in line with (Lebowitz et al., 2019)) that an indirect measure of the conformational state of DAT inside and outside the nanodomains could be obtained by parallel imaging of binding to DAT of a fluorescently tagged cocaine-analogue and of DAT itself; that is, as a result of its conformational bias the cocaine-analogue would be expected to bind more to outward-facing than to inward-facing transporters at a subsaturating concentration. We labeled cultured DA neurons and mEOS2-DAT expressing CAD cells with DG3-63 (10 nM), an analogue of cocaine tagged with $\mathrm{JF}_{646}$ suited for dSTORM (Figure 2A-D). The DG3-63 signal generally appeared somewhat less clustered as compared to that observed by our other approaches (see Figure 1). This might indicate a preferential binding of DG3-63 to unclustered DAT, which was supported by dual-color images of DG3-63 labelled DAT and mEOS-DAT, showing a more dispersed DG3-63 signal as compared to a more clustered mEOS2-DAT signal (Figure 2E-G). Importantly, the DG3-63 signal was specific as it was essentially eliminated by excess of unlabeled cocaine (Figure $2 \mathrm{H}$ ). To quantify whether DG3-63 preferentially bound to unclustered DAT, we used a modification of the principles outlined by the colocalization extension of the Voronoi tessellation algorithm (Levet et al., 2019) where associated DG3-63 was identified by including DG3-63 localizations within $25 \mathrm{~nm}$ from an mEOS2-DAT localization (Figure 2I-K). The total mEOS2-DAT Voronoi tessellated area distribution showed a preference for smaller tessellated areas consistent with a clustered distribution (Figure 2L). In contrast, the mEOS2-DAT-associated DG3-63 localizations resided on larger Voronoi tessellated areas; hence, DG3-63 binding was not enriched in the DAT nanodomains containing the highest density of DAT molecules (Figure 2L). This suggests that nanoclustered DAT are less prone to bind DG3-63, consistent with a more inward-facing transporter conformation or a less accessible binding site. Interestingly, by analyzing the distribution of mEOS2-DAT after treatment with an excess, and thereby saturating concentration, of cocaine, we observed a dispersal 
of the signal. This supports that forcing the transporter into an outward-facing conformation may release the transporter from the nanodomains (Figure S3).

We further rationalized that if the nanoscale DAT distribution reflects its molecular conformation, then a redistribution would be expected for a DAT variant with a conformational bias. Accordingly, we compared in transfected CAD cells clustering of WT DAT with that of a disease-associated DAT mutation (D421N) known to be shifted towards an inward-facing conformation (Hansen et al., 2014; Herborg et al., 2018) (Figure 2M-R). In agreement with the idea that inward-facing DAT will display preferential localization to nanodomains, dSTORM after immunolabeling revealed for D421N compared to WT both a higher fraction of large clusters (diameter $>75 \mathrm{~nm}$ ) and a higher fraction of localizations in dense clusters (Figure $2 \mathrm{~N}, \mathrm{O}, \mathrm{Q}, \mathrm{R}$ ) without a change in total number of detected localizations (Fig. 2P). Summarized, the data support that DAT preferentially assumes an inward-facing conformation in DAT nanodomains while the conformation outside the nanodomains conceivably is more outward-facing.

\section{NMDA receptor activation declusters DAT in striatal slices}

We next assessed whether DAT nanodomain distribution was sensitive to NMDA stimulation in intact tissue (Figure 2I-P) as we previously observed in cultured neurons (Rahbek-Clemmensen et al., 2017). Notably, NMDA receptors are present in DA terminals where they can stimulate DA release (Salamone et al., 2014). Acute coronal striatal slices were treated for $5 \mathrm{~min}$ with vehicle, NMDA or NMDA plus the NMDA receptor antagonist (2R)-amino-5-phosphonovaleric acid (AP5) before DAT immunolabeling and dSTORM (Figure 3A). Because each dSTORM image contained a vast number of DAT positive varicosities, we developed a new Voronoi tessellation-based collection algorithm for automated and unbiased isolation of these varicosities, allowing subsequent quantification of DAT clustering (Figure 3B, C). Following NMDA treatment, both the fraction of clusters $>75 \mathrm{~nm}$ and the faction of localizations in dense clusters decreased; an effect that was muted by the NMDA antagonist AP5 (Figure 3D, E). Note that the 
comparisons were normalized to the control sample from the individual imaging session to correct for unavoidable variability in sample preparations and between imaging sessions. Importantly, the data were further supported by Voronoi tessellation showing that NMDA, but not NMDA + AP5, reduced the fraction of smaller tessellated areas and thus clustering (Figure 3F). We conclude that local activation of NMDA receptors in intact tissue promotes dispersal of DAT nanodomains.

\section{DAT nanodomain localization is regulated by neuronal activity}

We speculated whether persistent changes in membrane potential and thereby in neuronal excitability would affect DAT nanodomain distribution. To test this, we expressed the bacterial sodium channel (mNaChBac) or the inward-rectifier potassium channel (Kir2.1) (Lin et al., 2010; Xue et al., 2014) in cultured DA neurons to make them more or less excitable, respectively (Lin et al., 2010). In agreement with selective expression in DA neurons, we confirmed that transduced cells were also DAT positive (Figure S4). Electrophysiological recordings on single neurons showed that the pacemaker firing activity of DA neurons (Rayport et al., 1992) was enhanced in mNaChBac-expressing neurons and reduced in Kir2.1 expressing neurons (Fig S4). Analysis by dSTORM of DAT distribution in mNaChBac expressing neurons showed decreased clustering, while for Kir2.1 expressing neurons we saw a trend towards an increased fraction of large clusters and a significant increase in localizations within large dense clusters (Fig. 4A-D). Thus, DAT nanodomain localization is in cultured DA neurons regulated by persistent changes in excitability or firing activity with increased activity leading to dispersal of DAT from nanodomains and decreased activity leading to increased nanodomains localization.

\section{DAT nanodomain localization is regulated by $\mathrm{Ca}^{2+}$}

Because of the effect of neuronal excitability, we decided to investigate putative involvement of $\mathrm{Ca}^{2+}$ influx via voltage-gated $\mathrm{Ca}^{2+}$ channels (VGCC) in NMDA-induced DAT declustering. Note that both P/Q 
type and N-type VGCCs should be present in DA terminals (Turner et al., 1993). DA neurons were stimulated with NMDA for 5 min with or without the N-type VGCC blocker $\omega$-conotoxin or the P/Q type VGCC blocker $\omega$-agatoxin. While we observed no effect of $\omega$-agatoxin, the dispersing effect of NMDA on DAT clustering was essentially abolished by $\omega$-conotoxin. Similarly, Voronoi tessellation analysis showed that $\omega$-conotoxin, but not $\omega$-agatoxin, blocked the NMDA-induced shift away from small, tessellated areas and thus blocked the dispersal of DAT nanodomains (Figure 4E-J, Movies S1-2). We next asked whether $\mathrm{Ca}^{2+}$ would be important per se by preincubating the neurons with the cell permeable $\mathrm{Ca}^{2+}$-chelator BAPTA-AM. Indeed, BAPTA-AM blunted the response to NMDA (Figure 4K-L). Of note, the pharmacological data fitted well with available single-cell mRNA sequencing data on DA neurons supporting highest transcription of N-type VGCC in DA neurons (Figure 4M). Taken together, NMDAinduced dispersal of DAT nanodomains appears to occur via $\mathrm{Ca}^{2+}$-dependent mechanism involving the activity of N-type VGCCs.

\section{DAT nanodomain localization is regulated by the D2 autoreceptor}

D2-autoreceptors in DA neurons act as negative feedback regulators decreasing neuronal excitability and firing activity by promoting opening of inhibitory G-protein-activated inwardly rectifying potassium channels (Beckstead et al., 2004; Kuzhikandathil et al., 1998). We surmised that D2-autoreceptor activity might regulate localization of DAT to nanodomains and tested this by determining the effect of the D2 receptor antagonist haloperidol and the D2 receptor agonist quinpirole. Interestingly, incubation with 10 $\mathrm{nM}$ haloperidol for $5 \mathrm{~min}$ resulted in dispersal of DAT nanodomains with a reduced fraction of clusters $>75 \mathrm{~m}$, fewer localizations in dense clusters and a reduced fraction of smaller Voronoi tessellated areas (Figure 5A-F, Movies S1, S3). This effect is likely the result of haloperidol blocking the effect on the D2autoreceptors of DA released in the culture as a consequence of DA neuronal pacemaker activity (Rayport et al., 1992). An alternative explanation would be that haloperidol blocks constitutive receptor activity 
(Strange, 2008). No matter what, co-incubation with an excess of quinpirole blocked the effect of haloperidol (Figure 5A-F). Moreover, the effect of haloperidol was accompanied by an increase in cytoplasmic free $\mathrm{Ca}^{2+}$ consistent with D2-autoreceptor activity suppressing neuronal firing (Figure S5). This prompted us to test if the effect of haloperidol, like the effect of NMDA, was dependent on $\mathrm{Ca}^{2+}$ influx via N-type VGCCs. This appeared to be the case as $\omega$-conotoxin, but not $\omega$-agatoxin, eliminated the effect of haloperidol (Figure 5A-F). Summarized, our data support that D2-autoreceptor activity can regulate DAT nanodomain localization via a $\mathrm{Ca}^{2+}$-dependent mechanism.

\section{Single particle tracking supports nanodomain dispersal in response to neuronal activity}

To obtain a better understanding of the dynamics underlying DAT nanodomain localization, we employed SPT-dSTORM (Manley et al., 2008) to assess if dispersal of DAT from nanodomains is accompanied by increased lateral mobility of DAT in the membrane. To allow visualization in live cells, we labeled DAT in DA neurons with a Janelia Fluor ${ }^{\circledR} 549$-conjugated cocaine analogue, DG3-80, suited for live dSTORM (Guthrie et al., 2020) (Figure 5G, H). Individual DAT tracks were captured with lengths up to $1.6 \mathrm{sec}$ (100 frames, 16 msec frame rate) with the majority being 10-20 frames (Figure 5I, J). Separating tracks into those reflecting confined movements and those reflecting freely moving particles showed that $90 \%$ of the tracks described freely moving particles (Figure $5 \mathrm{~K}, \mathrm{~L}$ ). Note that this distribution (with most of the labeled molecules being freely moving) likely reflects preferential binding of DG3-80 to unclustered DAT

in the outward-facing conformation (Figure 2). The mean jump distance (i.e. the distance an individual molecule/particle has traveled between each image) $\sim 110 \mathrm{~nm}$ was for all the recorded tracks and $\sim 50 \mathrm{~nm}$ for static tracks only (Figure 5M, N). Among the freely moving particles, however, some moved very fast with mean jump distance of 200-350 nm (Figure 5M-O). Interestingly, both NMDA receptor activation and blockade of the D2-autoreceptors with either haloperidol or the neutral antagonist UH-232 increased the population of DAT molecules with mean jump distances $>200 \mathrm{~nm}$, indicative of a possible link between 
dispersal of DAT nanodomains and increased lateral mobility. Note that the similar effect of UH-232 and haloperidol strongly suggest that their effect reflects blockade of D2-autoreceptors activated by DA, which is released because of DA neuronal pacemaker activity, and not inhibition of constitutive receptor activity.

\section{DAT nanodomains overlap with PIP2 nanodomains}

A key question is how the nanoscale distribution of DAT relates to other molecular components of DA terminals. We first turned to the plasma membrane phospholipid, PIP2, which can bind DAT and in doing so modulate DAT activity (Belovich et al., 2019). Dual-color dSTORM on PIP2 and DAT in DA neurons showed as expected (van den Bogaart et al., 2011), a distribution of the PIP2 signal into nanodomains (Figure 6). Strikingly, these nanodomains showed a remarkable overlap with DAT nanodomains with the Voronoi tessellation distributions revealing a large fraction of PIP2 localizations associated with dense DAT localizations (Figure 6A, B, F). As also seen for DAT domains, treatment with both NMDA and haloperidol reduced PIP2 localizations in large dense clusters while haloperidol also reduced cluster size. Importantly, all these changes were blocked by AP5 or quinpirole, respectively (Figure 6B-D). The NMDA and haloperidol-induced changes were reflected in the Voronoi tessellation distributions as well by showing dispersal of the PIP2 nanodomains and reduced PIP2 association to nanoclustered DAT (Figure $6 \mathrm{E}, \mathrm{F})$.

\section{The D2R is also shows nanodomain distribution}

We next analyzed the D2-autoreceptors that are found both in the somatodendritic compartment of DA neurons and in DA terminals. Due to known challenges with DA receptor antibodies, we first verified that our antibody was specific for the receptor in transfected HEK293 cells (Figure S5). Subsequent dual-color dSTORM experiments on cultured DA neurons revealed a clustered distribution of the D2-autoreceptors 
with nanodomains of a similar size as those found for DAT (Figure 7A, B). Furthermore, like our findings for DAT and PIP2, NMDA and haloperidol promoted dispersal of the receptor domains (Figure 7B-E). Relatively few of the nanodomains, however, overlapped with the DAT domains as apparent from the Voronoi tessellation distributions (Figure 7A, B, F), and as for D2-autoreceptor association to DAT, we only observed marginal changes upon NMDA and haloperidol treatment (Figure 7F).

The findings prompted us to further assess the specificity of the pharmacological manipulations by imaging additional key membrane components in DA terminals including clathrin (Maxfield and McGraw, 2004) and the single transmembrane SNARE protein syntaxin1 (STX-1). Imaging of clathrin revealed a nanoclustered distribution, possibly corresponding to clathrin-coated pits (Li et al., 2018), that showed little co-localization with DAT and, importantly, essentially no change in distribution in response to NMDA (Figure S6). We also recapitulated nanodomain distribution of STX-1 that is known to exist in such domains in presynaptic terminals (Padmanabhan et al., 2020) as well as the protein has been proposed to bind DAT (Binda et al., 2008). However, the STX-1 domains showed little overlap with DAT and responded differently to NMDA with a decreased fraction of large clusters and an increased fraction of localizations in dense clusters (Figure S6). In summary, several plasma membrane components of DA terminals show a nanoclustered distribution that, however, appears to be differentially regulated. 


\section{DISCUSSION}

DA neurons possess numerous presynaptic terminals or varicosities that serve as the principal architectural units responsible for release and reuptake of DA. Nonetheless, we still have a surprisingly poor understanding of the molecular organization of these varicosities, and what regulatory processes that might take place within these compartments that are less than $\sim 1 \mu \mathrm{m}$ in size. Here, we demonstrate that the application of super-resolution techniques enables detailed, quantitative analyses of how a key membrane protein, DAT, is subject to nanoscale regulation within these varicosities. This regulation involves $\mathrm{Ca}^{2+}$-dependent movements of DAT in and out of PIP2-enriched nanodomains in the plasma membrane, which are governed by NMDA receptor and D2-autoreceptor activity, and likely involves shift of DAT between outward and inward-facing conformations.

To firmly establish that DAT is distributed into nanodomains in the plasma membrane of DA neurons, we applied a range of different super-resolution microscopy techniques to both cultured neurons and striatal slices. A varied label density cluster analysis (Baumgart et al., 2016) supported that our dSTORM procedure reports true nanodomains, which was further confirmed by ExM, excluding potential artifacts from multiple countings of the same fluorophore. A rough estimate of the number of DAT molecules suggested the presence of 50-100 DAT molecules within each domain, which is in the same range estimated for AMPA receptor domains in the postsynapse ( 25 molecules) (Goncalves et al., 2020). Importantly, by using the novel fluorescent cocaine analogue DG3-63 and the disease-associated DAT mutant D421N, we were able to obtain evidence that DAT displays a conformational bias depending on whether it is present in a nanodomain or not. Based on these data, it is appealing to envision that a distribution of DAT between a nanoclustered, inward-facing population of transporter molecules and an unclustered, "free" outward-facing population of DAT molecules represents a means by which DAT activity can be regulated on a very fast time scale. A possible question is whether this dynamic localization of DAT to nanodomains involves changes in DAT oligomerization; however, we find this unlikely 
considering recent data supporting the existence in the plasma membrane of DA dimers that are highly stable even over the course of minutes (Das et al., 2019).

Of major importance, we validated in striatal slices the dispersing effect of NMDA on DAT nanodomains by employing a novel Voronoi tessellation-based collection algorithm. In addition to support that our findings in cultured DA neurons can be translated to intact tissue, the algorithm offers possibilities for future detailed, quantitative analyses of protein distribution in neuronal terminals in intact brain tissue. Another important observation was that both acute excitatory input, i.e. activation of NMDA receptors, and sustained alterations of neuronal excitability, as evidenced by expression of either mNaChBac or Kir2.1 (Lin et al., 2010; Xue et al., 2014) in DA neurons, affect the nanoscale distribution of DAT in presynaptic varicosities. We also observed a marked role of presynaptic D2R activity, further suggesting that the nanoscopic distribution of a membrane protein, such as DAT, is continuously adapted by several mechanisms to the immediate activity level of the neurons in which it is expressed. It may be considered that the effects of the pharmacological manipulations on DAT clustering are quantitatively modest. However, the effects are highly significant, as well as it should be considered that the numerous varicosities included in each quantification may be very heterogenous and possibly differentially sensitive to treatments. Such heterogeneity is directly supported by the finding that only a subset of DA terminals appeared active at any given time (Pereira et al., 2016). Future efforts should further clarify this interesting issue.

Our data revealed a striking co-localization of DAT and PIP2 nanodomains, as well as a dispersing effect of NMDA and haloperidol treatment on both types of domains. We also found that the effect of haloperidol and NMDA on DAT nanodomains not only required opening of VGCCs but also was contingent on the presence $\mathrm{Ca}^{2+}$. Interestingly, an increase in cytosolic $\mathrm{Ca}^{2+}$ was previously shown to release membrane protein sequestered PIP2, which might indicate a possible PIP2-dependent $\mathrm{Ca}^{2+}$-regulation of DAT nanodomain localization (McLaughlin and Murray, 2005). Indeed, the anionic lipid is known to form 
nanodomains that have been suggested to be important for membrane protein nanoclustering, including STX-1 (van den Bogaart et al., 2011). Also, PIP2 is known to regulate many cellular processes including ion channel and transporter function (Hamilton et al., 2014; Suh and Hille, 2008). Both the serotonin transporter (SERT) and DAT bind PIP2 (Buchmayer et al., 2013; Hamilton et al., 2014), and for DAT it was suggested that PIP2 binds to the DAT $\mathrm{N}$-terminus and induces a structural change that encourages phosphorylation and a reverse transport mode of DAT critical for amphetamine-induced DA efflux (Belovich et al., 2019; Hamilton et al., 2014). This is interesting in relation to our data that support an inward-facing configuration of nanodomain localized DAT and thereby presumably a more efflux prone transporter (Robertson et al., 2009). Of note, our previous study suggested a dependency on cholesterol for DAT nanodomain distribution (Rahbek-Clemmensen et al., 2017). Furthermore, previous data have indicated that cholesterol binds to DAT and regulates its function by promoting an outward-facing conformation (Hong and Amara, 2010). A conceivable explanation is that cholesterol serves to compartmentalize the membrane to enable a nanoclustered co-distribution of DAT and PIP2 (Carquin et al., 2016; Hwang et al., 1995), and accordingly that cholesterol rather interacts with unclustered, outwardfacing DAT. It is even possible that rapid movements of DAT in and out of nanodomains parallels the transport cycle shifting the transporter back and forth from an outward-facing, substrate binding, conformation and unclustered distribution to an inward-facing, substrate releasing (or efflux prone), conformation and clustered distribution.

Previous data have indicated that stimulation of D2-autoreceptors of both the D2 and D3 subtype enhance DAT activity (Bolan et al., 2007; Castro-Hernandez et al., 2015; Lee et al., 2007; Meiergerd et al., 1993; Parsons et al., 1993; Zapata et al., 2007). Interestingly, our finding that D2-autoreceptor activation promotes DAT nanoclustering may be consistent with such an enhancement; that is, if D2-autoreceptor activation causes an increase in DAT activity, this would expectably lead to a shift of the DAT conformational equilibrium towards a more inward-facing conformation and, hence, a likely shift towards 
a higher degree of nanodomain localization. However, our data may challenge that the regulation of DAT by D2-autoreceptors involves a direct interaction as previously suggested (Bolan et al., 2007; CastroHernandez et al., 2015; Lee et al., 2007). Although the D2-autoreceptor, like DAT, according to our dSTORM analyses, are distributed into nanodomains, we observed only modest co-localization between the two proteins in the DA terminals. The nanodomains responded, nonetheless, like DAT to NMDA and haloperidol, suggesting that excitatory input promotes nanodomain dispersal, while D2 autoreceptor activation promotes nanoclustering. It is tempting to speculate that this increased clustering upon activation reflects assembly of the receptor with intracellular signaling complexes and/or dynamics relating to desensitization and/or internalization.

DAT nanodomains showed little co-localization with nanodomains of STX-1 and clathrin that importantly also responded differently to NMDA. These differential effects support that the changes we see for DAT, the D2-autoreceptor and PIP2 most likely reflect true molecular redistributions and unlikely are the result of non-specific changes. Notably, the decrease in STX-1 nanocluster size and increased localization within dense clusters in response to NMDA is consistent with other studies indicating that breakup of STX-1 nanodomains, correlating with vesicle release, takes place, while at the same time more STX-1 is being brought to the sites of release (Maidorn et al., 2019; Padmanabhan et al., 2020). Of interest, the Voronoi tesselated distributions showed that NMDA slightly increased localization of STX1 to unclustered DAT (i.e. the larger tessellated areas). This suggests that the previously described direct interaction between DAT and STX-1, which have been suggested to be important for amphetamineinduced efflux (Binda et al., 2008), may take place between the unclustered fractions of the two proteins.

Summarized, by application of single-molecule sensitive super-resolution imaging we show compelling evidence that the molecular machinery supporting volume transmission mediated by a neuromodulator like DA is subject to spatial and temporal nanoscale regulation with putative major impact on synaptic function and homeostatic presynaptic plasticity. The conclusion is substantiated by 
detailed studies of DAT that according to our results is subject to remarkable nanoscale regulatory processes governed by excitatory input, membrane potential, $\mathrm{Ca}^{2+}$ and activity of the presynaptic D2 receptor. Taken together, the present results not only constitute a new framework for further deciphering the nanoscale architecture of presynaptic release sites and the underlying functional implications for the DA system but also add to the growing acknowledgment of nanoscopic regulation as a still poorly explored area of fundamental importance for neuronal signaling.

\section{ACKNOWLEDGEMENT}

We thank Ralph Götz for support with expansion microscopy. The work was supported by the Lundbeck Foundation grants R266-2017-4331 (UG), R276-2018-792 (UG), R230-2016-3154 (M.D.L.), R181-20143090 (FH), R303-2018-3540 (F.H.) and R231-2016-2481-5 (ATS), Independent Research Fund Denmark Medical Sciences (U.G. 7016-00325B), and the NIDA-Intramural Research Program Z1A DA000610 (AHN and DAG).

\section{AUTHOR CONTRIBUTIONS}

M.D.L. performed all the experiments and wrote the analyses not hereafter mentioned; A.L.E. devised the cluster size algorithm and identified mRNA expression data; A.T.S. performed electrophysiology and generated the construct encoding Cre recombinase under control of a truncated TH promotor; N.O.H. performed the D421N CAD cell experiments; S.H.J. cloned Kir2.1 and mNaChBac plasmids and made noncommercially available viruses; D.A.G. and A.H.N. invented and synthesized the fluorescent cocaine analogs; J.F.S. optimized the DA neuron culture protocol; M.D.L., F.H., and U.G. conceptualized the study, designed the research and interpreted data; U.G., F.H., M.S., and C.W. supervised the research. M.D.L., U.G., and F.H. wrote the paper with contributions from M.S. and all the other authors. 
bioRxiv preprint doi: https://doi.org/10.1101/2021.03.09.434538; this version posted March 10, 2021. The copyright holder for this preprint (which was not certified by peer review) is the author/funder. All rights reserved. No reuse allowed without permission.

\section{COMPETING FINANCIAL INTERESTS}

The authors declare no competing financial interests. 


\section{LEGENDS TO MAIN FIGURES}

Figure 1. DAT shows nanodomain distribution in cultured neurons and striatal slices

(A-D) Visualization of mEOS2-DAT overexpressed in cultured DA neurons. Endogenous TH was labeled to identify DA neurons. $(A, C)$ Widefield images ( $C$ is boxed region in $A)$. ( $B, D)$ PALM/dSTORM images (D is boxed region in B).

(E-H) 3D dSTORM image of DAT in DA neurons (color-coded based on depth). (E) Neuronal extension. (F) Varicosity (boxed region in E) (XY perspective). (G) YZ perspective. (H) XZ perspective.

(I-L) Mouse brain coronal section immunolabeled for DAT. (I) brain slice with striatal DAT staining. (J) Comparison of widefield with dSTORM. (K) Widefield view of varicosity. (L) dSTORM image.

(M-P) 10 x Expansion Microscopy (ExM) on mouse striatal slice immunolabeled for DAT. N shows region of interest (R.O.I.) in $\mathrm{M}$ with the full $19 \mu \mathrm{M} \mathrm{Z}$ slice (color-coded based on depth); O is R.O.I. in M with just $2 \mu \mathrm{M}$ of the $\mathrm{Z}$ axis. (P) Single varicosity identified in $\mathrm{O}$.

(Q-T) Cluster metrics demonstrated on a varicosity immunolabeled for DAT. Q, dSTORM of single varicosity. $(R, S)$ Identification of clusters by DBSCAN; clusters with diameter $>75 \mathrm{~nm}$ in green, clusters $<75 \mathrm{~nm}$ in magenta; dense clusters (>80 localizations within a radius of $50 \mathrm{~nm}$ ) in red. (T) DAT localizations segmented by Voronoi tessellation.

See also Figures S1 and S2.

Figure 2. Nanodomain localized DAT is preferentially in an inward-facing conformation

(A-D) dSTORM on DA neurons and DAT expressing CAD cells labeled with the fluorescent cocaine analogue DG3-63 (10 nM). (A) Axonal extension. (B) varicosity (box in A). (C) CAD cell. (D) R.O.I. from C.

(E-H) The density-based association of DG3-63 to mEOS2-DAT. (E) Cartoon of labeling strategy. (F) dSTORM/PALM image of CAD cell expressing mEOS2-DAT (red) and labeled with DG3-63 (10 
nM)(blue). (G) Close-up dSTORM/PALM image of CAD cell expressing mEOS2-DAT (red) and labeled with DG3-63 (10 nM) (blue). (H) The DG3-63 signal is blocked by $100 \mu \mathrm{M}$ cocaine.

(I-K) Voronoi tessellation based colocalization algorithm. Tessellation is performed on the mEOS2-DAT data and areas are marked for when the mEOS2-DAT localization has a DG3-63 localization within $25 \mathrm{~nm}$ distance.

(L) Left, Total mEOS2-DAT Voronoi tesselated area distribution (red) (probability density function (P.D.F.) and tessellated areas of mEOS2-DAT associated with DG3-63 (blue). Right, tessellated areas for mEOS2-DAT associated with DG3-63 (blue) compared to that associated in presence of saturating cocaine.

(M-O) TIRF/dSTORM of WT DAT and inward-facing mutant (D421N). (M) Example image of DAT expressed in CAD cells. (N) Example images of clusters with diameter $>75 \mathrm{~nm}$ (green) in WT DAT and D421N Dcells. (O) Normalized fraction of clusters with diameter $>75 \mathrm{~nm}$ (in \%), means \pm S.E., from 38 WT DAT cells and 42 D421N cells, 3 transfections, ${ }^{* * *} p<0.001$, unpaired t-test.

(P-O) dSTORM of WT DAT and inward-facing mutant (D421N). (P) Number of localizations per $\mu^{2}{ }^{2}$ for WT DAT and D421N, means \pm S.E., unpaired t test, $p=0.4$. (Q) Example image of dense clusters (red) of WT DAT and D421N (radius $100 \mathrm{~nm},>100$ localizations). (R) Fraction of localizations in dense clusters (in \% of WT DAT), means \pm S.E., from 38 WT DAT cells and 42 D421N cells, 3 transfections, $* * p<0.01$, unpaired t-test.

See also Figure S3.

\section{Figure 3. NMDA receptor activation declusters DAT in mouse brain slices}

(A) Workflow diagram.

(B) Data processing steps to isolate single varicosities from dSTORM images (see Methods).

(C) Representative DAT dSTORM image before, during, and after varicosity identification. 
(D) Example varicosity from control, NMDA, and AP5 + NMDA treated slices (clusters with diameter $>75 \mathrm{~nm}$ in green, clusters $<75 \mathrm{~nm}$ in magenta). Right, Normalized fraction of DAT clusters $>75 \mathrm{~nm}$ (in \%), means \pm S.E., from 3 experiments with analysis of 1793 control, 1102 NMDA and 1033 NMDA + AP5 varicosities; one-way ANOVA, $* * * * p<0.0001, * p<0.05$

(E) Example varicosity from control, NMDA $(20 \mu \mathrm{M})$, and AP5 + NMDA $(20 \mu \mathrm{M})$ treated slices (dense clusters in red, $>80$ localizations, radius $50 \mathrm{~nm}$ ). Right, Normalized fraction of DAT localizations in dense clusters (in \%), means \pm S.E.; one-way ANOVA, ${ }^{* * * *} p<0.0001, * * * p<0.001,{ }^{*} p<0.01$.

(F) The probability density functions (P.D.F.) for the Voronoi tessellated areas averaged by varicosity.

\section{Figure 4. DAT nanodomain localization is regulated by neuronal activity in a $\mathrm{Ca}^{2+}$ dependent manner}

(A-D) Expression of the $\mathrm{Na}^{+}$channel mNaChBac or the $\mathrm{K}^{+}$channel Kir2.1 in DA neurons. Example varicosities from DAT dSTORM of control, mNaChBac or Kir2.1 expressing DA neurons. (A) DAT clusters with diameter $>75 \mathrm{~nm}$ in green, clusters $<75 \mathrm{~nm}$ in magenta. (B) Dense DAT clusters in red (>80 localizations, radius $50 \mathrm{~nm}$ ). (C) Normalized fraction of clusters $>75 \mathrm{~nm}$ (in \%). (D) Normalized fraction of DAT localizations in dense clusters (in \%), means \pm S.E.; data from 3 cultures and 80 control, $230 \mathrm{mNaChBac}$ and 161 Kir2.1 varicosities; one-way ANOVA, ${ }^{* * * *} \mathrm{p}<0.0001,{ }^{* *} \mathrm{p}<0.01$, ${ }^{*} p<0.05$, n.s., not significant.

(E-J) The effect of NMDA on DAT nanodomains is blocked by the N-type VGCC blocker $\omega$-conotoxin. Example varicosities from dSTORM of DA neurons subject to 5 min treatment with vehicle, NMDA $(20 \mu \mathrm{M})$, AP5 $(100 \mu \mathrm{M})+$ NMDA $(20 \mu \mathrm{M})$, w-Conotoxin $(1 \mu \mathrm{M})+$ NMDA $(20 \mu \mathrm{M})$ or $\omega$-Agatoxin (1 $\mu \mathrm{M})+\mathrm{NMDA}(20 \mu \mathrm{M})$. (E) DAT clusters with diameter $>75 \mathrm{~nm}$ in green, clusters $<75 \mathrm{~nm}$ in magenta. (F) Dense DAT clusters in red (>80 localizations, radius $50 \mathrm{~nm}$ ). (G) localizations segmented by Voronoi tessellation. $(\mathrm{H}, \mathrm{I})$ Normalized fraction of DAT clusters $>75 \mathrm{~nm}$ (in \%) $(\mathrm{H})$ and normalized fraction of DAT localizations in dense clusters (in \%) (I), means \pm S.E.; one-way ANOVA, $* * * * p<$ 
$<0.0001,{ }^{*} p<0.05$, n.s., not significant. (J) Probability density functions (P.D.F.) (curves color-coded as in the bar diagrams) for the Voronoi tessellated areas averaged by varicosity. Data are from 3 cultures and 190 control, 147 NMDA, 170 AP5, $180 \omega$-Conotoxin and $154 \omega$-Agatoxin varicosities,

(K, L) Pretreatment (30 in) with BAPTA-AM inhibits the dispersing effect of NMDA on DAT nanodomains. (K) Normalized fraction of DAT clusters $>75 \mathrm{~nm}$ (in \%) and (L) normalized fraction of DAT localizations in dense clusters (in \%) for control, NMDA $(20 \mu \mathrm{M})$ and NMDA $(20 \mu \mathrm{M})+$ BAPTA-AM $(25 \mu \mathrm{M})$, means \pm S.E.; data from 3 cultures and includes analysis of 66 control, 72 NMDA and 70 BAPTA-AM + NMDA varicosities, one-way ANOVA, ${ }^{* *} p<0.01,{ }^{*} p<0.05$, n.s., not significant.

(M) mRNA expression for P, R, and N-type VGCC in DA neurons based on single cell RNAseq data (see Methods).

See also Figure S4 and Movies S1-2.

Figure 5. DAT nanodomain localization is regulated by the D2R and involves changes in lateral mobility

A-F) Dispersal of DAT nanodomains is promoted by the D2R antagonist haloperidol and blocked by the agonist quinpirole and by the N-type VGCC blocker $\omega$-conotoxin. Example varicosities from dSTORM of DA neurons subject 5 min treatment with haloperidol (10 nM), haloperidol (10 nM) + quinpirole $(50 \mu \mathrm{M})$, haloperidol $(10 \mathrm{nM})+\omega$-conotoxin $(1 \mu \mathrm{M})$ or haloperidol $(10 \mathrm{nM})+\omega$-agatoxin $(1 \mu \mathrm{M})$. (A) DAT clusters with diameter $>75 \mathrm{~nm}$ in green, clusters $<75 \mathrm{~nm}$ in magenta. (B) Dense DAT clusters in red (>80 localizations, radius $50 \mathrm{~nm}$ ). (C) localizations segmented by Voronoi tessellation. (D, E) Normalized fraction of DAT clusters $>75 \mathrm{~nm}$ (in \%) (D) and normalized fraction of DAT localizations in dense clusters (in $\%)(E)$, means \pm S.E.; one-way ANOVA, ${ }^{* * * * p} p<0.0001,{ }^{* * *} p<0.001$, ${ }^{* *} p<0.01,{ }^{*} p<0.05$, n.s., not significant. (F) Probability density functions (P.D.F.) (curves colorcoded as in the bar diagrams) for the Voronoi tessellated areas averaged by varicosity. Probability density functions (P.D.F.) (curves color-coded as in the bar diagrams) for the Voronoi tessellated 
areas averaged by varicosity. Data are from 3 cultures and 153 control, 166 haloperidol, 167 quinpirole, $178 \omega$-conotoxin and $176 \omega$-agatoxin varicosities,

(G-R) Single particle tracking dSTORM (SPT-dSTORM) identifies changes in DAT mobility in response to NMDA and D2R antagonism. (G) Example live dSTORM images of DA neurons labeled with the fluorescent cocaine analogue, DG3-80. (H) Boxed region in B. (I) Detected tracks and (J) Distribution of segment lengths in varicosity from control SPT-dSTORM experiment (16 ms frame rate). (K) Example traces of a confined and an unconstrained freely moving particle (DG3-80 labeled DAT). (L) Fraction of unconstrained tracks out of the total number of tracks. Data are means of 23 tracks \pm S.E. (M) Probability Density Function (P.D.F.) for total mean jump distances (M.J. D) (i.e. the distance an individual molecule/particle has traveled between each image) for control experiments. (N) P.D.F. when separated into static and freely moving particles. Among the freely moving particles, some moved very fast with M.J.D. of 200-350 nm. (O) NMDA (20 $\mu \mathrm{M}, 5 \mathrm{~min}$ ) increases population of DAT molecules with M.J.D. > $200 \mathrm{~nm}$, which is blunted by AP5 (100 $\mu \mathrm{M})$. (P) Fraction of fast moving particles (> $200 \mathrm{~nm}$ ), means \pm S.E., data from 13 control, 4 NMDA and 5 NMDA+AP5 experiments; $(\mathrm{Q}, \mathrm{R})$ The D2R antagonists haloperidol (10 nM, $5 \mathrm{~min}$ ) and UH-232 (1 $\mu \mathrm{M}$ ) increase the population of DAT molecules with M.J.D. $>200 \mathrm{~nm}>200 \mathrm{~nm}$; M, Fraction of fast moving particles (> $200 \mathrm{~nm}$ ), means \pm S.E., data are from 10 control, 6 haloperidol and $5 \mathrm{UH}-232$ experiments; one-way ANOVA, ${ }^{*} p<0.05 ;{ }^{*} p<0.01$, n.s., not significant.

See also Figure S5 and Movies S1, S3.

\section{Figure 6. DAT nanodomains overlap with PIP2 nanodomains}

(A) Example dual-color dSTORM images of phosphatidylinositol 4,5-bisphosphate (PIP2) (red, CF568) and DAT (blue, Alexa647) in a neuronal extension from cultured DA neurons (left) with close-up image of varicosity (right) corresponding to boxed region in the left image. 
(B) Example dual-color dSTORM images of varicosities from DA neurons showing localizations for PIP2 (red) and DAT (blue). Left, Control; Right, neurons exposed to haloperidol (10 nM, $5 \mathrm{~min}$ ).

(C-F) Effect of NMDA and haloperidol on PIP2 clustering. Upper panels, Control, NMDA (20 $\mu \mathrm{M})$ and NMDA $(20 \mu \mathrm{M})+$ AP5 $(100 \mu \mathrm{M}) ;$ Lower panels; Control, haloperidol (10 $\mathrm{nM})$ and haloperidol (10 $\mathrm{nM})+$ quinpirole $(50 \mu \mathrm{M}) .(\mathrm{C})$ Normalized fraction of PIP2 localizations in large clusters (>75 $\mathrm{nm}$ in diameter) (in \%) and (D), normalized fraction of PIP2 localizations in dense clusters (>80 localizations, radius $50 \mathrm{~nm}$ ) (in \%), means \pm S.E., one-way ANOVA, $* * * * p<0.0001, * * p<0.01$, n.s., not significant. (E) Probability density functions (P.D.F.) (color-coded as in the bar diagrams) for the Voronoi tessellated areas averaged by varicosity. (F) Probability density functions (P.D.F.) (colorcoded as in the bar diagrams) of the association of PIP2 with DAT clusters determined by Voronoi tessellation-based association (see Figure 2) showing strong association PIP2 with DAT. Data are from 3 cultures and 137 control, 126 NMDA, 121 NMDA + AP5 varicosities; and 140 control, 102 haloperidol, 67 haloperidol + quinpirole varicosities,

\section{Figure 7. The $D 2 R$ is also distributed into nanodomains}

(A) Example dual-color dSTORM images of the D2R (red, CF568) and DAT (blue, Alexa647) in DA neuronal extension (left) with close-up image of varicosity (right) corresponding to boxed region in the left image.

(B) Example dual color dSTORM images of varicosities from DA neurons showing localizations for D2R (red) and DAT (blue). Left, Control; Right, neurons exposed to haloperidol (10 nM, $5 \mathrm{~min}$ ).

(C-F) Effect of NMDA and haloperidol on D2R clustering. Upper panels, Control, NMDA (20 $\mu \mathrm{M})$ and NMDA $(20 \mu \mathrm{M})+$ AP5 $(100 \mu \mathrm{M})$; Lower panels; Control, haloperidol (10 $\mathrm{nM})$ and haloperidol (10 $\mathrm{nM})+$ quinpirole $(50 \mu \mathrm{M})$. (C) Normalized fraction of D2R localizations in large clusters (>75 $\mathrm{nm}$ in diameter) (in \%) and (D), normalized fraction of D2R localizations in dense clusters (>80 
localizations, radius $50 \mathrm{~nm}$ ) (in \%), means \pm S.E., one-way ANOVA, ${ }^{* * *} p<0.001,{ }^{* *} p<0.01,{ }^{*} p<0.05$, n.s., not significant. (E) Probability density functions (P.D.F.) (color-coded as in the bar diagrams) for the Voronoi tessellated areas averaged by varicosity. (F) Probability density functions (P.D.F.) (color-coded as in the bar diagrams) of the association of D2R with DAT clusters determined by Voronoi tessellation-based association (see Figure 2). Data are from 3 cultures and data from 3 cultures and 112 control, 157 NMDA, 75 NMDA + AP5; and 179 control, 195 haloperidol and 150 quinpirole varicosities.

See also Figure $\mathrm{S} 5$ and $\mathrm{S} 6$. 


\section{LEGENDS TO SUPPLEMENTARY FIGURES}

\section{Figure S1. Estimation of protein copy number in DAT clusters (Related to Figure 1.)}

(A) To estimate how many DAT molecules that are present in an average DAT nanodomain defined by the DBSCAN analysis, we used a protocol modified from (Ehmann et al., 2014; Siddig et al., 2020) and labeled cultured DA neurons with a twofold dilution row (1:3200-1:100) of primary Mab369 DAT antibody, followed by labeling with Alexa647-conjugated secondary antibody and dSTORM imaging. The figures show four example varicosities labeled with lowest level of primary DAT antibody (1/3200 dilution). Individual nanodomains/clusters are shown by separate colors. For each cluster the number of localizations was counted, as shown by the histogram to right of each example.

(B) The clustering analysis was performed on 85 varicosities labeled with 1/3200 dilution of primary antibody and the total number of localizations per cluster for the entire set is shown in the left panel. The graph indicates that the minimum number of localizations in a cluster as a conservative estimate is 3 at this extreme antibody dilution and thus that 3 localizations likely may arise from a single primary antibody in this set of images. We next counted the number of localizations found in varicosities labeled with increasing concentrations of primary antibody $(1 / 100,1 / 200,1 / 400$, $1 / 800,1 / 1600,1 / 3200$ as indicated by color) and counted the number of localizations present within clusters that has a diameter greater than $75 \mathrm{~nm}$ (right panel). The resulting distributions, shown color-coded, peaked at an estimated 100 localizations. It follows that estimated $\sim 33$ copies of the monocolonal antibody would be present that in turn each can label up to two DAT molecules. Accordingly, a rough estimate says that an average cluster contains 50-100 DAT molecules.

(C) Example varicosities showing DAT localizations in green in clusters with a diameter larger than 75 nm using a 1/100 dilution of primary DAT antibody. 
Figure S2. Cluster analysis by varied label density cluster verification (Related to Figure 1.)

(A) Example dSTORM images of varicosities from DA neuronal cultures labeled with DAT primary antibody at indicated dilutions.

(B) Localization present in clusters $(\rho / \rho 0)$ was compared to the relative area of the ROI covered by a cluster mask (n), with each data point being a single varicosity (Baumgart et al., 2016). The red line indicates a reference curve from a random distribution and thus the data strongly support a true clustered distribution of DAT localizations into nanodomains and accordingly that the clustering of the DAT signal observed is not the result of multiple observations of single fluorophores in agreement with our previous data in DAT expressing CAD cells (Rahbek-Clemmensen et al., 2017).

Figure S3. Cocaine promotes declustering of DAT in transfected CAD cells (Related to Figure 2).

(A) Example PALM/TIRF images of the mEOS2 DAT expressed in plasma membrane of CAD cell with and without incubation with an excess of cocaine. The experiment was done while examining DG363's association with unclustered DAT where unlabeled cocaine was applied to the CAD cells to compete with DG3-63 signal and to show its specificity. The excess cocaine caused a declustering of DAT.

(B) Probability density function (P.D.F.) for the Voronoi tessellated areas averaged by varicosity showing a dramatic reduction in the clustering of DAT following cocaine application. Data from 16 control cells ( 3 transfections) and 17 treated cells ( 2 transfections).

Figure S4. Overexpression in DA neurons of $\mathrm{mNaChBac}$ increases while Kir2.1 decreases neuronal excitability (Related to Figure 4).

(A-C) Verification of selective expression of bacterial ion channels in DA neurons by a dual viral transduction protocol. The cultured DA neurons were co-infected with an AAV encoding either Cre- 
dependent mNaChBac or Kir2.1 and with an AAV encoding Cre recombinase under control of a truncated tyrosine hydroxylase (TH) promoter (AAV-pTH-iCre-WPREpA) (see Methods). Images show a putative Kir2.1 expressing DA neuron immunostained for the co-expressed protein mKate2 (red) and for DAT (blue). (A) Widefield image with merged DAT and Kir2.1 signal; B, Kir2.1 signal; C, DAT signal.

(D) Whole-cell patch-clamp of a DA neuron expressing Kir2.1. Merged image showing IR-DIC and mKate2 signal of a DA neuron and a patch electrode approaching the cell soma.

(E) Left, Current-clamp recording mode (at $0 \mathrm{pA}$ ) showing increased firing activity of mNaChBac expressing DA neuron (purple) and essentially no firing activity of Kir2.1 expressing DA neuron (black) as compared to control neuron (green). Right, Action potential frequency $(\mathrm{Hz})$ in control $(n=6), m N a C h B a c(n=4)$ and Kir2.1 $(n=5)$ expressing neurons, means \pm S.E.; one-way ANOVA with Dunnett's multiple comparison test, ${ }^{* *} \mathrm{p}<0.01$.

Figure S5. The effect of the D2R antagonist haloperidol cytosolic $\mathrm{Ca}^{2+}$ in terminals of cultured DA neurons and verification of D2R antibody (Related to Figure 5).

(A) Live imaging of terminals from DA neurons expressing the genetically $\mathrm{Ca}^{2+}$ sensor jRGECO (three example neurons shown). Right images are enlarged from boxed regions shown in left images. The neurons are, as indicated, shown both immediately before and $200 \mathrm{sec}$ after exposure to 10 nM haloperidol.

(B) Averaged fluorescence jRGECO signal over time measured for 6 presynaptic terminals showing how haloperidol (10 $\mathrm{nM})$ cause a rapid rise in cytosolic $\mathrm{Ca}^{2+}$.

(C) Verification of D2R antibody. HEK293 cell were transiently transfected with the D2R short isoform or DAT. Widefield microscopy show D2R immunosignal in some but not all HEK 293 cells transiently 
expressing the D2R short isoform. No signal was observed in DAT expressing HEK cells stained with D2R antibody.

\section{Figure S6. Analysis of STX-1 and clathrin nanodomains (Related to Figures 6 and 7).}

(A) Example dual-color dSTORM images of syntaxin-1 (STX-1) (red, CF56) and DAT (blue, Alexa647) (upper left panels), and clathrin (red, CF56) and DAT (blue, Alexa647) (lower left panels) in DA neuronal extension (left) with close-up of varicosity (right) corresponding to boxed region in left image.

(B) Example dual-color dSTORM images of varicosities from DA neurons showing localizations for syntaxin-1 (STX-1) (red, CF56) and DAT (blue, Alexa647) (upper right panels), and clathrin (red, CF56) and DAT (blue, Alexa647) (lower right panels), Left, Control; Right, neurons exposed to NMDA (20 $\mu \mathrm{M}, 5 \mathrm{~min})$.

(C-J) Effect of NMDA on STX-1 and clathrin clustering. Cultured DA neurons were stimulated with vehicle (control), NMDA $(20 \mu \mathrm{M})$ or NMDA $(20 \mu \mathrm{M})+$ AP5 $(100 \mu \mathrm{M})$ before DAT and STX-1/clathrin immunolabeling for dSTORM analysis. (C, D) Normalized fraction of STX-1 or clathrin localizations in large clusters ( $>75 \mathrm{~nm}$ in diameter) (in \%) and (E, F), normalized fraction of STX-1 or clathrin localizations in dense clusters (>80 localizations, radius $50 \mathrm{~nm}$ ) (in $\%$ ), means \pm S.E., one-way ANOVA, ${ }^{* *} p<0.01,{ }^{*} p<0.05$, n.s., not significant. (G, H) Probability density functions (P.D.F.) (colorcoded as in the bar diagrams) for the Voronoi tessellated areas averaged by varicosity (G, STX-1; H, Clathrin). (I, J) Probability density functions (P.D.F.) (color-coded as in the bar diagrams) of the association of of STX-1 or clathrin with DAT clusters determined by Voronoi tessellation-based association (see Figure 2). Data are from 3 cultures and 217 control, 278 NMDA, 183 NMDA + AP5 varicosities (STX-1); and 98 control, 98 NMDA, 108 NMDA + AP5 varicosities (clathrin). 
Figure S7. Validation of the multicolor alignment of our microscope (Related to Star Methods).

To verify the multicolor alignment of our microscope, dSTORM images of $100 \mathrm{~nm}$ Tetraspek beads were taken. Localizations were fit to widefield illuminated Tetraspec beads and processed as our dSTORM data is processed. Localization data was generated into images as such to show how well aligned the multicolor signal is in our system.

\section{LEGENDS TO SUPPLEMENTARY MOVIES}

Movie S1. 3D-STORM of DA neuronal varicosity immunostained for DAT (control conditions) (Related to Figures 4 and 5). Color-code is based on density where red localizations are defined as over 300 localizations within $100 \mathrm{~nm}$ radius, white as $\sim 150$ localizations within a $100 \mathrm{~nm}$ radius, and the remainder in blue.

Movie S2. 3D-STORM representation of DA neuronal varicosity immunostained for DAT (5 min NMDA treatment) (Related to Figures 4 and 5). Color-code is based on density where red localizations are defined as over 300 localizations within $100 \mathrm{~nm}$ radius, white as 150 localizations within a 100 $\mathrm{nm}$ radius, and the remainder in blue.

Movie S3. 3D-STORM representation of DA neuronal varicosity immunostained for DAT (5 min haloperidol treatment) (Related to Figure 5). Color-code is based on density where red localizations are defined as over 300 localizations within $100 \mathrm{~nm}$ radius, white as $\sim 150$ localizations within a $100 \mathrm{~nm}$ radius, and the remainder in blue. 


\section{STAR*METHODS}

\section{LEAD CONTACT}

Further information and requests for resources and reagents should be directed to and will be fulfilled by the Lead Contact, Ulrik Gether (gether@sund.ku.dk)

\section{MATERIALS AVAILABILITY}

All unique reagents generated in this study are available from the Lead Contact. Plasmids generated are available upon request.

\section{DATA AND CODE AVAILABILITY}

Custom written codes used for the data analysis can be found at: https://github.com/GetherLab/SuperResolution-Data-Analysis. . The datasets supporting the current study have not been deposited in a public repository because of the file size but are available from the corresponding author on request.

\section{EXPERIMENTAL MODEL AND SUBJECT DETAILS}

\section{Primary cultures of DA neurons with cortical glia cells}

Cultured glial cells were obtained from cortex and cultured DA neurons were obtained from the midbrain of P1-P3 Wistar rats (Charles River, Germany). Gender was mixed as the cultures were generated from a mixed sex populations of rat pups. For preparation of cultured glial cells, brains were removed from euthanized P1-P3 pups and placed in phosphate-buffered saline (PBS) on ice. The cortex of each brain was isolated and cut into millimeter sized sections and collected in cold dissection medium (Hanks' Balanced Salt Solution with added Sodium Pyruvate (1 mM), Penicillin-Streptomycin (Sigma P0781, 200 U/L), 10 mM HEPES, and $0.54 \%$ glucose)). After collecting all, the dissection medium was replaced with glia cell medium (DMEM with HEPES "1965", FBS (10\%), Penicillin-Streptomycin (60 U/L)). The tissue was 
mechanically titrated through pipetting up and down using a flame-polished Pasteur pipette followed by straining through a $70 \mu \mathrm{m}$ cell trainer and centrifugation for $10 \mathrm{~min}$ at $200-300 \mathrm{x}$. The pellet was resuspended in $10 \mathrm{~mL}$ glia medium and strained again before a second centrifugation for $10 \mathrm{~min}$ at 200$300 \mathrm{~g}$ and removal of the supernatant. The pellet was resuspended and seeded into large T175 flasks at approximately 1.5 brains per flask with added glial medium to a total of $20 \mathrm{~mL}$. Glia cells were grown in an incubator at $37 \circ \mathrm{C}$ with $5 \% \mathrm{CO}_{2}$ until they were $70 \%$ confluent. The cells were either frozen in heatinactivated serum containing $10 \%$ DMSO for later use or applied to ethanol-cleaned and poly-D-lysinecoated coverslips and incubated for 1 week prior to DA neuron addition. On the day before DA neuron addition, the glial cells were changed to DA neuron medium (Neurobasal A with heat-inactivated FBS (1/100), GlutaMAX (1/100) B-27 (1/50), ascorbic acid $(200 \mu \mathrm{M})$, Penicillin-Streptomycin $(60 \mathrm{U} / \mathrm{L})$, kynurenic acid (0.5 mM)). Midbrain DA neurons were generated using a protocol modified from (Rayport et al., 1992). Brains from rat pups (P1-P3) were isolated and placed in PBS on ice. Midbrain sections were retrieved from each brain as the brains were cut while in dissection buffer. The midbrain sections were transferred to papain solution (cysteine $(1 \mathrm{mM})$, DNase $(0.1 \mathrm{mg} / \mathrm{mL})$, papain $(20$ units $/ \mathrm{mL}), \mathrm{NaCl}(116$ mM), $\mathrm{KCl}(5.4 \mathrm{mM}), \mathrm{CaCl}_{2}(1.9 \mathrm{mM}), \mathrm{NaHCO} 3(26 \mathrm{mM}), \mathrm{NaH}_{2} \mathrm{PO}_{4} \mathrm{H}_{2} \mathrm{O}(2 \mathrm{mM}), \mathrm{MgSO}_{4}(1 \mathrm{mM}), \mathrm{EDTA}$ (0.5 $\mathrm{mM})$, Glucose $(25 \mathrm{mM})$, kynurenic acid $(0.5 \mathrm{mM})$ in water) for $30 \mathrm{~min}$ at $37{ }^{\circ} \mathrm{C}$. The solution was replaced with prewarmed DA medium and samples were mechanically titrated with flame polished Pasteur pipettes and the resulting suspension centrifuged at $200-300 \times \mathrm{g}$ for $10 \mathrm{~min}$. The pellet was resuspended in DA neuron medium and applied to each culture. Glia-derived neurotrophic factor (GDNF) (100ng/mL of culture solution volume) was applied immediately after seeding of the neurons. Cultures were incubated for 2 to 3 weeks prior to use. All procedures were performed according to institutional guidelines at the Faculty of Health Sciences, University of Copenhagen. 


\section{Mouse brain slices}

Mouse brain slices were obtained from male C57BL/6 mice 12-16 weeks old. The slices were prepared as described in Methods Details. All procedures were performed according to institutional guidelines at the Faculty of Health Sciences, University of Copenhagen.

\section{Heterologous cell culture}

Cath.a-differentiated (CAD) cells (Qi et al., 1997) (ATCC CRL-11179 or Sigma-Aldrich 08100805), originating from mouse (B6/D2 F1 hybrid) catecholaminergic neuronal tumor. The cells were maintained in a 1:1 mixture of DMEM 1965 and Ham's F-12 medium (Invitrogen), both supplemented with 10\% FBS and $0.01 \mathrm{mg} / \mathrm{mL}$ gentamicin. HEK293 cells (human embryonic kidney cells, ATCC CRL-1573) were grown as described (Hansen et al., 2014).

\section{METHOD DETAILS}

\section{Molecular biology, virus generation and virus transduction}

The cDNA encoding human DAT (synthetic gene kindly provided by Dr. Jonathan Javitch, Columbia University, NY, USA (Loland et al., 2004) was inserted into pmEOS2-C1 (RRID: Addgene\#54510) to generate pmEOS2-hDAT C1 encoding hDAT with mEOS2 fused to the N-terminus. Plasmids encoding WT hDAT and D421N hDAT ( $p R C / C M V$ hDAT and pRC/CMV hDAT D421N) were generated as described (Herborg et al., 2018)). The cDNA encoding the short transcriptional variant of human DA D2 receptor with an N-terminal FLAG tag (SF-D2R-S) was inserted into mammalian expression vector pcDNA 3.1(+) (Invitrogen) (Klewe et al., 2008). The Cre-dependent construct, pAAV-CAG-Flex-NES-jRGECO1a-WPREpA, was obtained from Addgene (RRID: Addgene \#100854). The plasmids, pAAV-hSyn-DIO-mNaChBac-T2AmKate2-WPREpA and pAAV-hSyn-DIO-Kir2.1-T2A-mKate2-WPREpA, were SLIC cloned (Jeong et al., 2012) from pAAV-EF1 $\alpha$-F-FLEX-mNaChBac-T2A-tdTomato (RRID: Addgene \#60658) and pAAV-EF1 $\alpha$-F-FLEX- 
Kir2.1-T2A-tdTomato (RRID: Addgene \#60661), respectively, into a Cre-dependent AAV backbone plasmid with a mKate2 fluorophore instead of tdTomato. This was done to make them sufficiently short so that packaging into an AAV capsid would be feasible (ITR to ITR length<4700 kbp). The pAAV-pTH-iCreWPREpA construct was created and cloned in-house and contains a truncated $488 \mathrm{bp}$ rat TH promoter encoding Cre recombinase. In-house generated AAVs were produced using a FuGene6 (Promega) mediated triple plasmid co-transfection method in HEK293t cells. Three days after transfection, cells were harvested and virus purified using an adapted lodixanol gradient purification protocol (Matsui et al., 2012). Genomic AAV titer was determined by a PicoGreen-based method as described elsewhere (Piedra et al., 2015). AAV2-retro-pTH-iCre mediated expression of Cre-dependent jRGECO1 was verified by fluorescence microscopy and no differences were observed across the different titers used. For transduction, the appropriate virus was applied to primary culture DA neurons at an approximate concentration of $2 * 10 \mathrm{E} 9 \mathrm{vg} / \mathrm{mL}$ at 1 to 3 days after preparation of the neuronal cultures.

\section{Transfection of DA neurons}

Primary cultures of DA neurons were transfected using Magnetotransfection at 14 DIV (Underhill et al., 2014). NeuroMag reagent (OZ biosciences) $(8 \mu \mathrm{L})$ was mixed with plasmid (4 $\mu \mathrm{g})$ and DA neuron medium $(200 \mu \mathrm{L})$. The mixture was incubated for $20 \mathrm{~min}$ at RT before it was added to cultures grown in 6-well plates. The culture was positioned on top of a magnetic plate for $15 \mathrm{~min}$ after which the culture before it was moved back to the cell incubator and used for immunostaining 24-48 hours later.

\section{Immunostaining of primary cultures of DA neurons for DSTORM and PALM}

The cell samples were fixed in paraformaldehyde (3\%) and washed three times in glycine $(20 \mathrm{mM})$ and $\mathrm{NH}_{4} \mathrm{Cl}(50 \mathrm{mM})$ in PBS. Subsequently, the cells were washed in blocking buffer ( $5 \%$ Donkey serum, $1 \% \mathrm{BSA}$ in PBS), and incubated in blocking permeabilization buffer (blocking buffer with saponin (0.2\%)). Primary 
antibody was applied in blocking buffer for $60 \mathrm{~min}$, followed by 3-5 min incubation in blocking buffer. Secondary antibody was applied in blocking buffer for $45 \mathrm{~min}$, and the sample was incubated $2 \mathrm{x}$ for $5 \mathrm{~min}$ in blocking buffer. Samples were washed in PBS twice and post-fixated in paraformaldehyde (3\%) for 15 min. Samples were washed twice in glycine $(20 \mathrm{mM})$ and $\mathrm{NH}_{4} \mathrm{Cl}(50 \mathrm{mM})$ in PBS and stored in $\mathrm{PBS}$ at $4^{\circ} \mathrm{C}$ until imaging.

\section{Preparation and immunostaining of mouse brain slices for dSTORM}

Mice were anesthetized with isoflurane and perfused first with PBS followed by $4 \%$ paraformaldehyde (PFA) injected directly into the heart. Brains were then placed in 4\% PFA for 24 hours, followed by sucrose (30\%) for 24 to 48 hours. Subsequently, brains were snap-frozen on powdered dry ice and stored at $80^{\circ} \mathrm{C}$. The brains were sliced using a cryostat (Leica CM3050 S) to obtain $10 \mu \mathrm{m}$ striatal coronal sections and each slice was immediately fused to a 3-aminopropyltriethoxysilane coated coverslip and let dry at room temperature before being stored in antifreeze (50\% glycerol $50 \%$ PBS) at $-20 \circ \mathrm{C}$. For immunostaining, brain sections were first rinsed $3 x$ in PBS and subsequently washed 2x with 15 min incubation in glycine $(20 \mathrm{mM})$ and $\mathrm{NH}_{4} \mathrm{Cl}(50 \mathrm{mM})$ in PBS. This was followed by 30 min wash in $10 \mathrm{mM}$ trisodium citrate, $\mathrm{pH} 6.0$, preheated to $80^{\circ} \mathrm{C}, 4 \mathrm{x}$ wash in PBS and then blocking/permeabilization for 30 min in PBS with 5\% donkey serum, 1\% BSA and 0.3 \% Triton X-100. Primary antibody was applied overnight in blocking/permeabilization buffer followed by washing in 5/5/90 min increments and application of secondary antibody overnight in blocking/permeabilization buffer. Sections were again washed in 5/5/90 minute increments in blocking/permeabilization buffer and then with 5/5 min increments with PBS. The sections were post-fixated in paraformaldehyde (3\%) and washed twice for $15 \mathrm{~min}$ in glycine $(20 \mathrm{mM})$ and $\mathrm{NH}_{4} \mathrm{Cl}(50 \mathrm{mM})$ in PBS. Samples were lastly washed twice in PBS and stored in PBS at $4{ }^{\circ} \mathrm{C}$ until imaging. 


\section{Antibodies and labeling}

Secondary antibodies (donkey anti-rat, Jackson 712-005-153, RRID:AB_2340631; donkey anti-mouse, Jackson 715-005-151, RRID:AB_2340759); or donkey anti-rabbit, Jackson 711-005-152, RRID:AB_2340585) were labeled with either NHS-ester conjugated Alexa647 or CF ${ }^{\mathrm{TM}} 568$ (Biotium, Freemont, CA) and then isolated through Zeba ${ }^{\mathrm{TM}}$ Spin Desalting Columns, 40K MWCO (ThermoFisher 87767). This was completed by first washing the column $3 x$ with $300 \mu \mathrm{L}$ of $100 \mathrm{mM} \mathrm{NaHCO}$ in $\mathrm{PBS}$. Following the washes, $50 \mu \mathrm{g}$ of antibody in $100 \mu \mathrm{L}$ of PBS was added and spun at $1500 \mathrm{xg}$ for 2 mins. The flow-through was collected and combined with a 5-fold molar excess of NHS ester fluorophore. This solution was incubated in the dark at RT, shaking, for 2.5 hours. A new spin column was then washed 3 times with NaN--3 (0.02\%) in PBS. The antibody sample with the dye was then added to the column and centrifuged for $2 \mathrm{~min}$ at $1500 \mathrm{xg}$. The protein concentration and label concentration of the final product were measured on a NanoDrop 2000 Spectrophotometer (ThermoFisher). Labeled antibodies had between 1 and 2 fluorophores attached.

We used the following antibodies and fluorophores for our imaging analysis:

\begin{tabular}{|c|c|c|c|c|c|}
\hline $\begin{array}{l}\text { Antibody } \\
\text { Target }\end{array}$ & Product Number & Experiment & Dilution & $\begin{array}{l}\text { Secondary } \\
\text { antibody }\end{array}$ & Fluorophore \\
\hline DAT & $\begin{array}{l}\text { Millipore MAB369 } \\
\text { RRID:AB_2190413 }\end{array}$ & $\begin{array}{l}\text { Primary culture } \\
\text { dSTORM }\end{array}$ & $1 / 200$ & $\begin{array}{c}5 \mu \mathrm{g} / \mathrm{mL} \text { Donkey } \\
\text { anti-Rat }\end{array}$ & Alexa647 \\
\hline DAT & $\begin{array}{l}\text { Millipore MAB369 } \\
\text { RRID:AB_2190413 }\end{array}$ & IHC dSTORM & $1 / 200$ & $\begin{array}{c}10 \mu \mathrm{g} / \mathrm{mL} \\
\text { Donkey anti-Rat }\end{array}$ & Alexa647 \\
\hline DAT & $\begin{array}{l}\text { Millipore MAB369 } \\
\text { RRID:AB_2190413 }\end{array}$ & Expansion (ExM) & $1 / 200$ & $\begin{array}{c}5 \mu \mathrm{g} / \mathrm{mL} \text { Donkey } \\
\text { anti-Rat }\end{array}$ & Atto $643 \mathrm{~N}$ \\
\hline $\mathrm{TH}$ & $\begin{array}{l}\text { Millipore MAB318 } \\
\text { RRID:AB_2201528 }\end{array}$ & PALM/dSTORM & $1 / 500$ & $\begin{array}{c}5 \mu \mathrm{g} / \mathrm{mL} \text { Donkey } \\
\text { anti-Mouse }\end{array}$ & Alexa647 \\
\hline STX-1 & $\begin{array}{l}\text { Synaptic Systems } 110 \\
011 \text { RRID:AB_887844 }\end{array}$ & $\begin{array}{l}\text { Primary culture } \\
\text { dSTORM }\end{array}$ & $1 / 500$ & $\begin{array}{c}5 \mu \mathrm{g} / \mathrm{mL} \text { Donkey } \\
\text { anti-Mouse }\end{array}$ & CF568 \\
\hline $\begin{array}{c}\text { Clathrin } \\
\text { light } \\
\text { chain }\end{array}$ & $\begin{array}{l}\text { Synaptic Systems } 113 \\
011 \text { RRID:AB_887706 }\end{array}$ & $\begin{array}{l}\text { Primary culture } \\
\text { dSTORM }\end{array}$ & $1 / 500$ & $\begin{array}{c}5 \mu \mathrm{g} / \mathrm{mL} \text { Donkey } \\
\text { anti-Mouse }\end{array}$ & CF568 \\
\hline PIP2 & $\begin{array}{l}\text { Abcam ab11039 } \\
\text { RRID:AB_442848 }\end{array}$ & $\begin{array}{l}\text { Primary culture } \\
\text { dSTORM }\end{array}$ & $1 / 500$ & $\begin{array}{c}5 \mu \mathrm{g} / \mathrm{mL} \text { Donkey } \\
\text { anti-mouse }\end{array}$ & CF568 \\
\hline
\end{tabular}




\begin{tabular}{|c|c|c|c|c|c|}
\hline $\mathrm{D} 2 \mathrm{R}$ & $\begin{array}{l}\text { Synaptic Systems } 376 \\
203 \text { RRID:AB_2636918 }\end{array}$ & $\begin{array}{l}\text { Primary culture } \\
\text { dSTORM }\end{array}$ & $1 / 500$ & $\begin{array}{c}5 \mu \mathrm{g} / \mathrm{mL} \text { Donkey } \\
\text { anti-rabbit }\end{array}$ & CF568 \\
\hline mKate2 & $\begin{array}{c}\text { Evrogen AB233 } \\
\text { RRID:AB_2571743 }\end{array}$ & $\begin{array}{l}\text { Primary culture } \\
\text { dSTORM }\end{array}$ & $1 / 500$ & $\begin{array}{l}5 \mu \mathrm{g} / \mathrm{mL} \text { Donkey } \\
\text { anti-rabbit }\end{array}$ & CF568 \\
\hline
\end{tabular}

\section{Expansion Microscopy (ExM)}

For ExM, perfused brains were acquired by injecting first PBS and then $4 \%$ PFA into the heart of anesthetized mice. Brains were postfixated in 4\% PFA for 24 hours and immersed in sucrose solution (30\%) for 24 to 48 hours. Coronal brain slices $(40 \mu \mathrm{m})$ were obtained with a cryostat. The brain slices were stored, free floating, in antifreeze (50\% glycerol $50 \%$ PBS) at $-20{ }^{\circ} \mathrm{C}$ until use. Brain slices were washed with PBS three times, and then permeabilized in blocking buffer ( $5 \%$ donkey serum, $1 \%$ bovine serum albumin, $0.1 \%$ Triton X-100, in PBS). Brain slices were incubated overnight with primary antibody in blocking buffer ( $5 \%$ donkey serum, $1 \%$ bovine serum albumin, $0.1 \%$ Triton $\mathrm{X}-100$, in PBS, pH 7.4). Slices were washed $4 \mathrm{x}$ in blocking buffer with 30 min increments and incubated overnight with secondary antibody in blocking buffer at $4{ }^{\circ} \mathrm{C}$. Slices were again washed $4 \mathrm{x}$ in blocking buffer with 30 min increments. Anchoring treatment was performed with Acryloyl-X-SE $(0.1 \mathrm{mg} / \mathrm{mL})$ in PBS at room temperature overnight (Truckenbrodt et al., 2019). Gel solution was made by mixing N,N-dimethylacrylamide (1.335 g) and sodium acrylate $(0.32 \mathrm{~g})$ in $2.85 \mathrm{~g}$ of water. Oxygen was purged with nitrogen gas for $40 \mathrm{~min} .2 .7 \mathrm{~mL}$ of the gelling solution were mixed with $0.3 \mathrm{~mL} 0.036 \mathrm{~g} / \mathrm{mL}$ solution of potassium persulfate $\left(\mathrm{K}_{2} \mathrm{~S}_{2} \mathrm{O}_{8}\right)$ solution and purged of oxygen with nitrogen gas for 15 min on ice. Brain slices were transferred to the extra gelling solution and placed flat on poly-D-Lysine coated glass slides. . Excess gelling solution was removed, and glass coverslips were placed on the edges of each slice to limit gel dispersion. All work from this point was done in a cold room $(4 \circ \mathrm{C})$. Aliquots of $500 \mu \mathrm{L}$ of polymerization solution were combined with $2 \mu \mathrm{L}$ of TEMED was added and the solution vortexed before applying $80 \mu \mathrm{L}$ to brain slices. A layer of parafilm covered coverslip was placed overtop of the gel to fully surround the gel. The sample was kept 
in the cold room for 15 min before being brought back out to room temperature. The container with the gels was filled with nitrogen and wet paper to keep humidity and made airtight. Following gelation, samples were carefully cut out from the chamber in a sim-card shape to ensure orientation. Gels were measured in size, and placed in digestion buffer overnight at room temperature (50mM TRIS, $800 \mathrm{mM}$ guanidine $\mathrm{HCl}, 2 \mathrm{mM} \mathrm{CaCl} 2,0.5 \%$ Triton $\mathrm{X}-100$, proteinase $\mathrm{K} 8 \mathrm{U} / \mathrm{mL}, \mathrm{pH}$ 8.0). The gels were placed into individual dishes and dialyzed with water many times over a day until they no longer expanded. Imaging was performed the next day on a spinning disk confocal microscope (FEl CorrSight utilizing a CApochromat 63x/1.20 W Corr M27 objective). Samples were places dry on poly-D-Lysine coated chambers and water was added to the top of the gel just enough so the gel was hydrated but not so it would move around.

\section{dSTORM}

For dSTORM we used a buffer containing $\beta$-mercaptoehtanol and an enzymatic oxygen scavenger system (10\% (w/V) glucose, 1\% (V/V) beta-mercaptoethanol, 2 mM cyclooctatetraene, $50 \mathrm{mM}$ Tris- $\mathrm{HCl}(\mathrm{pH} 8), 10$ $\mathrm{mM} \mathrm{NaCl}, 34 \mu \mathrm{g} \mathrm{mL}^{-1}$ catalase, $28 \mu \mathrm{g} \mathrm{mL}^{-1}$ glucose oxidase). The imaging was performed with an ECLIPSE Ti-E epifluoresence/TIRF microscope (NIKON, Japan) equipped with $405 \mathrm{~nm}, 488 \mathrm{~nm}$ 561, and $647 \mathrm{~nm}$ lasers (Coherent, California, USA). All lasers are individually shuttered and collected in a single fiber to the sample through a 1.49 NA, 100x, apochromat TIRF oil objective (NIKON). Single color imaging was done with a dichroic mirror ( $(405 / 488 / 561 / 647 \mathrm{rpc}$ ) and the emitted light was filtered by a $710 / 80 \mathrm{~nm}$ bandpass filter. For dual-color dSTORM, we used a dichroic mirror with the range 350-412, 485-490, 558-564, and 637-660 nm (97,335 QUAD C-NSTORM C156921). The excitation light was filtered at the wavelengths: $401 \pm 24 \mathrm{~nm}, 488 \pm 15 \mathrm{~nm}, 561 \pm 15 \mathrm{~nm}, 647 \pm 24 \mathrm{~nm}$. The emitted light was filtered at the wavelengths: $425-475,505-545,578-625$, and $664-787 \mathrm{~nm}$, and secondly by an extra filter to decrease noise (561 $\mathrm{nm}$ Longpass, Edge Basic, F76-561, AHF). A motorized piezo stage controlled by a near-infrared light-adjusted 
perfect focus system (NIKON) is applied to the system to reduce any sample drift over time in the zdirection. Single-color dSTORM images were constructed from 30,000 frames taken at a 16 ms frame rate. Dual-color dSTORM images were constructed from 20,000 frames for each color taken at a 16 ms frame rate with each color alternating by frame. Photons were collected with an iXon3 897 EM-CCD camera camera (Andor, United Kingdom). Laser powers used were $2.3 \mathrm{~kW} \mathrm{~cm} \mathrm{-2} \mathrm{for} 647 \mathrm{~nm}, 1.0 \mathrm{~kW} \mathrm{~cm}-2$ for $488 \mathrm{~nm}$ and for $561 \mathrm{~nm}$. The $405 \mathrm{~nm}$ laser was used to incrementally increase blinking behavior at power $<0.1 \mathrm{~kW} \mathrm{~cm}-2$. For 3D-dSTORM, a cylindrical lens was placed before the camera to impart astigmatism.

\section{PALM}

For PALM imaging of mEos-DAT, we obtained 5,000 consecutive frames with a frame rate of $33 \mathrm{~Hz}$ to construct one image. The $488 \mathrm{~nm}$ excitation laser was held constant, $0.4 \mathrm{~kW} \mathrm{~cm}^{-2}$, during the capture of the image while the $405 \mathrm{~nm}$ activation laser was gradually increased to $<0.1 \mathrm{~kW} \mathrm{~cm}^{-2}$.

\section{Localization fitting of dSTORM data}

2D localizations were fit with ThunderSTORM using local maximum detection with a threshold of 1.5 ${ }^{*}$ std(Wave.F1) and estimator of an integrated gaussian PSF. Sigma was 1.6, fit radius was 3 pixels, and weighted least squares was the method to fit the estimated gaussian to the PSF (Ovesny et al., 2014). 3D localization fitting was performed with fit3Dcspline (Li et al., 2018). 3D calibrations were made on $100 \mathrm{~nm}$ TetraSpeck beads attached to a coverslip by first placing $50 \mu \mathrm{L}$ of $\mathrm{MgCl} 2(1 \mathrm{M})$ on the coverslip and then adding $500 \mathrm{~L}$ of water with $0.9 \mu \mathrm{L}$ of TetraSpeck beads to the coverslip. Drift for all data was corrected with redundant cross correlation through Matlab (Wang et al., 2014). Localizations were filtered for uncertainty being less than $25 \mathrm{~nm}$ and then merged for localizations that were detected within $15 \mathrm{~nm}$ and 3 frames. 


\section{Data processing pipeline - dSTORM}

To perform necessary localization fitting on the 1349 dSTORM images included in this study, an in-house data processing pipeline was developed to streamline operations. Localizations were fit to the raw data with the aforementioned settings in ThunderSTORM through a self-written ImageJ macro. Although ThunderSTORM can perform the remaining processing steps, a custom python script (utilizing Matlab functions) was written to perform cross correlation, merging and filtering so that redundant cross correlation could be employed. The images were then blinded by labeling them with a random integer and saving the cypher elsewhere. Through a separate custom Matlab script, varicosities were selected from each image though a freehand selection. Varicosities were identified by the local swelling of their structure compared to the rest of the extension. We next used DBSCAN (density based spatial clustering of applications with noise) (Ester et al., 1996; Rahbek-Clemmensen et al., 2017) to identify clusters by size or for determining localizations in dense clusters through the use of a python script employing the DBSCAN method from the sklearn python library. Voronoi tessellation (Levet et al., 2019) was employed through Matlab. The Voronoi based density association algorithm was also written in house and using Matlab.

\section{Varicosity identification in dSTORM DA neuron culture data}

Automatic varicosity identification could not discern between extension and varicosity, so these were selected through a freehand ROI taken of blinded files. Localization data for all images in a given dataset were blinded by being randomly assigned a number that was stored on a cypher. ROls were then gathered without the knowledge of the image identity, with this information restored in the analysis of the ROIs. 


\section{Varicosity identification in brain slice dSTORM data}

As this was a computationally intensive process, each image was divided into 100 quadrants in a $10 \times 10$ grid. Within each quadrant, the localizations were sub-sampled down to 1000 random localizations, and the Voronoi tessalation was obtained for that section. Tesselated areas were filtered for having an area less than $15000 \mathrm{~nm}^{2}$. Tesselated shapes that were touching one another were merged together. Shapes were now filtered for possessing an area greater than $300000 \mathrm{~nm}^{2}$. These ROIS were buffered by $50 \mathrm{~nm}$ along each edge to smooth the ROI.

\section{Clustering analysis of dSTORM data}

Cluster size was identified by performing DBSCAN on each varicosity for a radius of $15 \mathrm{~nm}$ and 5 localizations being the cluster qualifier. The convex hull was gathered for each cluster to find its area, and the fraction of clusters with a diameter $>75 \mathrm{~nm}$ was used for the cluster size analysis. Cluster size distributions were not shown as clusters reaching the detection limit of this method are not accurate and cause a false perception on the cluster size distribution. Localizations in dense clusters were found by applying DBSCAN to each varicosity with a radius of $50 \mathrm{~nm}$ and 80 localizations being the qualifier. Voronoi tessellation was applied to each varicosity and areas were filtered for sizes between $5 \mathrm{~nm} 2$ and $1000 \mathrm{~nm} 2$. Probability density functions were created for these distributions.

\section{Density Association}

Density association was found by performing Voronoi tessellation on target $A$, and finding all points in target $B$ that were $25 \mathrm{~nm}$ from any point in target $A$. Each of these target B points that possessed A association were mapped to the Voronoi tessellated area from target $A$ they resided in. The probability density functions for target $A$ and the areas from target $A$ that had an associated target B were created. 
This procedure was applied to compare the dSTORM signal of DG3_63 to mEOS2-DAT, STX1 to DAT, D2R to DAT, PIP2 to DAT, and clathrin to DAT.

\section{Quantification of DAT molecules in nanodomains}

Primary DA neurons were labeled and imaged for dSTORM with the DAT MAB369 antibody at dilutions 1:200, 1:400, 1:800, 1:1600, 1:3200. Varicosities were manually selected from blinded data files. Clusters of localizations potentially arising from single localizations were identified in the varicosities found in the 1:3200 dilution data set through DBSCAN with the parameters $[\varepsilon=30 \mathrm{~nm}, \mathrm{nps}=2]$. A histogram was made comparing the number of localizations found per cluster from this cluster analysis, and 3 localizations was determined to be the estimate for the number of localizations arising from a single monoclonal antibody in this image set. Clusters were then found in the remaining dataset through the same method defined to identify cluster size. A histogram was made to show the number of localizations present within the clusters found with a diameter $>75 \mathrm{~nm}$. The peak of this histogram was $\sim 100$ localizations, suggesting there are roughly 33 primary antibodies labeling DAT within a given cluster/nanodomain of this size.

\section{Varied label density cluster verification}

Varicosities used for the counting experiment were subject to the varied label density cluster verification test (Baumgart et al., 2016). Sig for gaussians was set to $15 \mathrm{~nm}$ and the cut gauss at $\mathrm{x}$ times sig was set to 2. The threshold selected was 2 .

\section{Labeling and imaging primary cultures with DG3-63}

Live primary cultures were labeled with DG3-63 (20 nM) (Guthrie et al., 2020) for 10 min in the dark at room temperature and washed 3 times with aCSF ( $\mathrm{NaCl}(120 \mathrm{mM}), \mathrm{KCl}(5 \mathrm{mM}), \mathrm{CaCl}_{2}(2 \mathrm{mM}), \mathrm{MgCl}_{2}(2$ mM), $1 \mathrm{mM} \mathrm{NaH} \mathrm{PO}_{4}$, HEPES (25 mM), glucose (30 mM), pH 7.4). Samples were fixed with 
paraformaldehyde (3\%) for 15 min followed by 3 washes with glycine $(20 \mathrm{mM})$ and $\mathrm{NH}_{4} \mathrm{Cl}(50 \mathrm{mM})$ in PBS. Samples were imaged in normal dSTORM buffer.

\section{Transfection of CAD cells and labeling with DG3-63}

CAD cells were transfected with pmEOS2-hDAT encoding mEOS-DAT or with pRC/CMV-hDAT or pRC/CMVhDAT D421N encoding WT DAT and the DAT mutant D421N, respectively (Herborg et al., 2018). The CAD cells ( $\sim 1$ million cells) were seeded the day before transfection in a $25 \mathrm{~cm}^{2}$ flask and washed on the day of transfection before addition of $5 \mathrm{~mL}$ of medium. For the transfection, $1 \mu \mathrm{g}$ of DNA was added to 100 $\mu \mathrm{L}$ of optiMEM as well as $3 \mu \mathrm{L}$ Lipofectamine (Invitrogen) to a separate $100 \mu \mathrm{L}$ of optiMEM. After $5 \mathrm{~min}$, the two solutions were mixed and incubated for $30 \mathrm{~min}$ before the total mixture was added to the cells. The next day the cells were harvested and seeded on poly-D-Lysine coated coverslips $(100,000$ cells per coverslip). For DG3-63 labeling experiments, the media was removed the next day from the mEOS-DAT expressing cells and $1 \mathrm{~mL}$ of aCSF was added ( $\mathrm{NaCl}(120 \mathrm{mM}), \mathrm{KCl}(5 \mathrm{mM}), \mathrm{CaCl}_{2}(2 \mathrm{mM}), \mathrm{MgCl}_{2}(2 \mathrm{mM})$, HEPES ( $25 \mathrm{mM})$, glucose (30 mM), pH 7.4) before incubation for $10 \mathrm{~min}$ with or without $100 \mu \mathrm{M}$ unlabeled cocaine. Following this, the cells were labeled with 10 nM DG3-63 (Guthrie et al., 2020) for 10 min in aCSF. Samples were washed 3 times with aCSF and then fixed for $15 \mathrm{~min}$ in paraformaldehyde (3\%) followed by 2x wash with glycine $(20 \mathrm{mM})$ and $\mathrm{NH}_{4} \mathrm{Cl}(50 \mathrm{mM})$ in PBS. Samples were imaged in dSTORM buffer as described above. For dSTORM of hDAT WT and D421N, cells the cover slips samples were stained in the same fashion as primary DA cultures.

\section{Pharmacological treatment of acute brain slices}

Mice were euthanized with isoflurane and the live brains were removed. Coronal sections, $300 \mu \mathrm{m}$ thick, were cut with a vibratome in cold aCSF ( $\mathrm{NaCl}(119 \mathrm{mM}), \mathrm{KCl}(2.5 \mathrm{mM}), \mathrm{NaH}_{2} \mathrm{PO}_{4}(24 \mathrm{mM})$, Glucose (12.5 $\left.\mathrm{mM}), \mathrm{CaCl}_{2}(2 \mathrm{mM}), \mathrm{MgCl}_{2}(2 \mathrm{mM})\right)$ that was being aerated with carbogen gas. Brain slices were 
transferred to aCSF and maintained for 1 hour at room temperature under constant carbogenation. Slices were then transferred to either aCSF or aCSF with AP5 $(100 \mu \mathrm{M})$, both at $37 \circ \mathrm{C}$ for $10 \mathrm{~min}$. Slices from normal aCSF were moved to aCSF with or without NMDA $(20 \mu \mathrm{M})$. The NMDA containing aCSF had reduced magnesium $(0.3 \mathrm{mM} \mathrm{MgCl} 2)$ and added glycine $(100 \mu \mathrm{M})$. The samples from AP5 were transferred to aCSF with AP5 $(100 \mu \mathrm{M})$ and NMDA $(20 \mu \mathrm{M})$, reduced magnesium $(0.3 \mathrm{mM} \mathrm{MgCl})$, and added glycine $(100 \mu \mathrm{M})$. These incubated for $5 \mathrm{~min}$, and then the slices were transferred to paraformaldehyde (3\%) overnight at $4 \circ \mathrm{C}$. Slices were transferred to a $30 \%$ sucrose solution for 2 days a $4{ }^{\circ} \mathrm{C}$ and then placed into plastic molds and covered with Tissue-Tek O.C.T. and stored at $-80{ }^{\circ} \mathrm{C}$. Samples were sliced at $10 \mu \mathrm{m}$ on the cryostat and stained for dSTORM.

\section{Pharmacological treatment of primary cultures}

In general, treatments were performed by replacing the media at room temperature with aCSF ( $\mathrm{NaCl}(120$ $\mathrm{mM}), \mathrm{KCl}(5 \mathrm{mM}), \mathrm{CaCl}_{2}(2 \mathrm{mM}), \mathrm{MgCl}_{2}(2 \mathrm{mM}), 1 \mathrm{mM} \mathrm{NaH} \mathrm{PO}_{4}, \mathrm{HEPES}(25 \mathrm{mM})$, glucose (30 mM), pH 7.4) followed by stimulation with indicated compounds. For the NMDA or haloperidol experiments with testing of VGCC inhibitors, cells were treated for 10 min with either control aCSF, AP5 (100 $\mu \mathrm{M})$ or quinpirole $(50 \mu \mathrm{M}), \omega$-conotoxin $(1 \mu \mathrm{M})$, $\omega$-agatoxin $(1 \mu \mathrm{M})$. Samples were then treated for 5 min with these same concentrations as cotreatment with either NMDA $(20 \mu \mathrm{M})$ or haloperidol $(10 \mathrm{nM})$. NMDA treatments were done in aCSF with added glycine $(100 \mu \mathrm{M})$. All experiments using NMDA were done with aCSF with reduced magnesium $\left(0.3 \mathrm{mM} \mathrm{MgCl}_{2}\right)$ for all conditions. For the BAPTA-AM experiment, the pretreatment with BAPTA-AM $(25 \mu \mathrm{M})$ was for 30 min prior to the 5-min NMDA $(20 \mu \mathrm{M})$ treatment.

\section{SPT-dSTORM}

Live primary cultures were labeled with DG3-80 (20 nM) for 10 min and then washed 3 times with aCSF ( $\mathrm{NaCl}(120 \mathrm{mM}), \mathrm{KCl}(5 \mathrm{mM}), \mathrm{CaCl}_{2}(2 \mathrm{mM}), \mathrm{MgCl}_{2}$ (2 mM-0.3 mM for NMDA related experiments), $1 \mathrm{mM}$ 
$\mathrm{NaH}_{2} \mathrm{PO}_{4}, \mathrm{HEPES}(25 \mathrm{mM})$, glucose $(30 \mathrm{mM}), \mathrm{pH}$ 7.4). A suitable location to image was found while the sample was kept in aCSF, and then SPTdSTORM imaging was done in a modified dSTORM buffer (560 $\mu \mathrm{g} / \mathrm{mL}$ glucose oxidase, $34 \mu \mathrm{g} / \mathrm{mL}$ catalase, $1.4 \mu \mathrm{L} / \mathrm{mL} \beta$-Mercaptoethanol in aCSF). Pharmacological treatment were done 5 min before imaging with either $20 \mu \mathrm{M}$ NMDA with $100 \mu \mathrm{M}$ glycine, $200 \mathrm{nM}$ haloperidol, or $1 \mu \mathrm{M}$ UH-232. AP5 $(100 \mu \mathrm{M})$ was applied for 10 min prior to cotreatment with NMDA. Imaging was performed at room temperature on the NIKON TiE Eclipse TIRF microscope described under dSTORM. Light with a wavelength of $561 \mathrm{~nm}$ at $0.3 \mathrm{~kW} \mathrm{~cm}^{-2}$ was utilized to promote blinking of the fluorophores. Images were collected with 16 ms frame rate. Localizations were fit using ThunderStorm as described above. Single molecule trajectories were found using the program swift (Turkowyd et al., 2020).

Parameter used beyond the default were: "expdisplacement" : 300,"expnoiserate": 10,"maxblinkingduration" : 2,"pblink" : 0.5, ,preappear" : 0.5, ,pswitch" : 0.001, ,precision": 30,"pruningbase" : 2,"pruningrate" : 0.2,"randomseed" : 42,"wdiffusion" : 2,"wdirected" : 1,"wfield" : 0, ,wimmobile" : 1

\section{Electrophysiology recordings of DA neurons}

DA neuronal cultures were transduced after 3 DIV (days in vitro) with pAAV-pTH-iCre-WPREpA plus either pAAV-hSyn-DIO-mNaChBac-T2A-mKate2-WPREpA or pAAV-hSyn-DIO-Kir2.1-T2A-mKate2-WPREpA. Electrophysiological recording by the whole-cell patch clamp technique were done 2-3 weeks after transduction. The DA neurons expressing either mNaChBac or Kir2.1 were identified based on mKate2 fluorescence using an upright microscope (Olympus BX51WI). For the recordings, the cultures were submerged in circulating, heated, and oxygenated aCSF (in mM; $\mathrm{NaCl} 125, \mathrm{KCl} 2.5, \mathrm{NaHCO}_{3} 26, \mathrm{CaCl}_{2} 2$, $\mathrm{MgCl}_{2} 1, \mathrm{NaH}_{2} \mathrm{PO}_{4} 1.25$, Glucose $\left.25 ; 2 \mathrm{ml} / \mathrm{min}, 35^{\circ} \mathrm{C}\right)$. The aCSF was supplemented with NBQX (20 $\left.\mu \mathrm{M}\right)$. The patch glass electrode was filled with (in mM) K-gluconate $122, \mathrm{Na}_{2}-\mathrm{ATP} 5, \mathrm{MgCl}_{2} 2.5, \mathrm{CaCl}_{2} 0.0003$, Mg-Gluconate 5.6, K-Hepes 5, H-Hepes 5, and EGTA 1, and had tip resistance of 4-6 M $\Omega$. After break-in, 
the cells were held at $0 \mathrm{pA}$ in current clamp mode to measure the pacemaker activity. Recordings were acquired with a Multiclamp 700B amplifier and 1440A Digitizer.

\section{Calcium Imaging of DA neurons}

Primary DA neurons were prepared as described above but instead of culturing the neurons on glass coverslips, they were cultured on MatTek $35 \mathrm{~mm} 14 \mathrm{~mm}$ glass microwell (no 1.5) glass bottom dishes (MatTek). To enable measurement of $\mathrm{Ca}^{2+}$-fluctuations specifically in DA neurons, the neurons were transduced with an AAV encoding double-floxed jRGECO1a (AAV-CAG-Flex-NES-jRGECO1a-WPREpA) together with an AAV encoding Cre recombinase under control of a truncated TH promoter (AAV2-retropTH-iCre-WPREpA). The cell medium was changed to aCSF ( $\mathrm{NaCl}(120 \mathrm{mM}), \mathrm{KCl}(5 \mathrm{mM}), \mathrm{CaCl} 2(2 \mathrm{mM})$, $\mathrm{MgCl}_{2}(2 \mathrm{mM}), 1 \mathrm{mM} \mathrm{NaH} \mathrm{PO}_{4}$, HEPES (25 mM), glucose (30 mM), pH 7.4) immediately prior to imaging. Imaging took place at room temperature on the NIKON TiE Eclipse TIRF microscope described above. Once a suitable neuron was located, the sample was imaged with $561 \mathrm{~nm}$ light $\left(10 \mathrm{~W} \mathrm{~cm}{ }^{-2}\right)$. Videos were collected with a $16 \mathrm{~ms}$ frame rate. Haloperidol was added mid-video so that the sample had a total concentration of $10 \mathrm{nM}$ Haloperidol. Data was collected from three independent experiments. Two varicosities were identified per image.

\section{RNA-seq mining and data analysis}

All RNA sequencing data was accessed via the Gene Expression Omnibus (GEO) under the following accession numbers: GSE108020, GSE115070, GSE76381, GSE116470, GSE116138. Raw counts were converted to RPKM and quantile normalized using the preprocessCore (3.11) package in R. 


\section{Quantification and Statistical Analysis}

Statistical details can be found in the figure legends and were computed with GraphPad Prism 8. When employing one-way ANOVA, Dunnett's post hoc test was used for multiple comparisons. The dSTORM comparisons were normalized to the control sample from the individual imaging session. This was done to control for unavoidable variability in sample preparations and between imaging sessions.

\begin{tabular}{|c|c|c|c|}
\hline Experiment & $\begin{array}{l}\text { Number of sample } \\
\text { preparations }\end{array}$ & $\begin{array}{l}\text { Number of dSTORM } \\
\text { Images }\end{array}$ & $\begin{array}{l}\text { Number of varicosities } \\
\text { (neuron experiments) } \\
\text { or cells (CAD cell } \\
\text { experiment) }\end{array}$ \\
\hline IHC dSTORM NMDA & 3 & 102 & 4028 \\
\hline CAD Cell D421N & 3 & 55 & 80 \\
\hline Membrane Potential & 3 & 108 & 471 \\
\hline NMDA and VGCC & 3 & 188 & 841 \\
\hline Haloperidol and VGCC & 3 & 180 & 840 \\
\hline BAPTA-AM & 3 & 95 & 208 \\
\hline STX1 NMDA & 4 & 162 & 678 \\
\hline Clathrin NMDA & 3 & 90 & 304 \\
\hline PIP2 Haloperidol & 3 & 84 & 309 \\
\hline PIP2 NMDA & 3 & 88 & 384 \\
\hline D2 NMDA & 3 & 101 & 344 \\
\hline D2 Haloperidol & 3 & 90 & 524 \\
\hline
\end{tabular}




\section{REFERENCES}

Baker, S.M., Buckheit, R.W., 3rd, and Falk, M.M. (2010). Green-to-red photoconvertible fluorescent proteins: tracking cell and protein dynamics on standard wide-field mercury arc-based microscopes. BMC Cell Biol 11, 15.

Baumgart, F., Arnold, A.M., Leskovar, K., Staszek, K., Folser, M., Weghuber, J., Stockinger, H., and Schutz, G.J. (2016). Varying label density allows artifact-free analysis of membrane-protein nanoclusters. Nat Methods 13, 661-664.

Beckstead, M.J., Grandy, D.K., Wickman, K., and Williams, J.T. (2004). Vesicular dopamine release elicits an inhibitory postsynaptic current in midbrain dopamine neurons. Neuron 42, 939-946.

Belovich, A.N., Aguilar, J.I., Mabry, S.J., Cheng, M.H., Zanella, D., Hamilton, P.J., Stanislowski, D.J., Shekar, A., Foster, J.D., Bahar, I., et al. (2019). A network of phosphatidylinositol (4,5)-bisphosphate (PIP2) binding sites on the dopamine transporter regulates amphetamine behavior in Drosophila Melanogaster. Molecular Psychiatry.

Bermingham, D.P., and Blakely, R.D. (2016). Kinase-dependent Regulation of Monoamine Neurotransmitter Transporters. Pharmacol Rev 68, 888-953.

Betzig, E., Patterson, G.H., Sougrat, R., Lindwasser, O.W., Olenych, S., Bonifacino, J.S., Davidson, M.W., Lippincott-Schwartz, J., and Hess, H.F. (2006). Imaging intracellular fluorescent proteins at nanometer resolution. Science 313, 1642-1645.

Beuming, T., Kniazeff, J., Bergmann, M.L., Shi, L., Gracia, L., Raniszewska, K., Newman, A.H., Javitch, J.A., Weinstein, H., Gether, U., et al. (2008). The binding sites for cocaine and dopamine in the dopamine transporter overlap. Nat Neurosci 11, 780-789.

Binda, F., Dipace, C., Bowton, E., Robertson, S.D., Lute, B.J., Fog, J.U., Zhang, M., Sen, N., Colbran, R.J., Gnegy, M.E., et al. (2008). Syntaxin $1 A$ interaction with the dopamine transporter promotes amphetamine-induced dopamine efflux. Mol Pharmacol 74, 1101-1108.

Bjorklund, A., and Dunnett, S.B. (2007). Dopamine neuron systems in the brain: an update. Trends Neurosci 30, 194-202.

Bolan, E.A., Kivell, B., Jaligam, V., Oz, M., Jayanthi, L.D., Han, Y., Sen, N., Urizar, E., Gomes, I., Devi, L.A., et al. (2007). D2 receptors regulate dopamine transporter function via an extracellular signal-regulated kinases 1 and 2-dependent and phosphoinositide 3 kinase-independent mechanism. Mol Pharmacol 71, 1222-1232.

Borroto-Escuela, D.O., Perez De La Mora, M., Manger, P., Narvaez, M., Beggiato, S., Crespo-Ramirez, M., Navarro, G., Wydra, K., Diaz-Cabiale, Z., Rivera, A., et al. (2018). Brain Dopamine Transmission in Health and Parkinson's Disease: Modulation of Synaptic Transmission and Plasticity Through Volume Transmission and Dopamine Heteroreceptors. Front Synaptic Neurosci 10, 20.

Buchmayer, F., Schicker, K., Steinkellner, T., Geier, P., Stübiger, G., Hamilton, P.J., Jurik, A., Stockner, T., Yang, J.-W., Montgomery, T., et al. (2013). Amphetamine actions at the serotonin transporter rely on the availability of phosphatidylinositol-4,5-bisphosphate. Proceedings of the National Academy of Sciences of the United States of America 110, 11642-11647.

Carquin, M., D'Auria, L., Pollet, H., Bongarzone, E.R., and Tyteca, D. (2016). Recent progress on lipid lateral heterogeneity in plasma membranes: From rafts to submicrometric domains. Progress in Lipid Research 62, 1-24. 
Castro-Hernandez, J., Afonso-Oramas, D., Cruz-Muros, I., Salas-Hernandez, J., Barroso-Chinea, P., Moratalla, R., Millan, M.J., and Gonzalez-Hernandez, T. (2015). Prolonged treatment with pramipexole promotes physical interaction of striatal dopamine D3 autoreceptors with dopamine transporters to reduce dopamine uptake. Neurobiol Dis 74, 325-335.

Chen, F., Tillberg, P.W., and Boyden, E.S. (2015). Optical imaging. Expansion microscopy. Science 347, 543-548.

Choquet, D., and Hosy, E. (2020). AMPA receptor nanoscale dynamic organization and synaptic plasticities. Curr Opin Neurobiol 63, 137-145.

Das, A.K., Kudlacek, O., Baumgart, F., Jaentsch, K., Stockner, T., Sitte, H.H., and Schutz, G.J. (2019). Dopamine transporter forms stable dimers in the live cell plasma membrane in a phosphatidylinositol 4,5-bisphosphate-independent manner. J Biol Chem 294, 5632-5642.

Descarries, L., Watkins, K.C., Garcia, S., Bosler, O., and Doucet, G. (1996). Dual character, asynaptic and synaptic, of the dopamine innervation in adult rat neostriatum: a quantitative autoradiographic and immunocytochemical analysis. J Comp Neurol 375, 167-186.

Ehmann, N., van de Linde, S., Alon, A., Ljaschenko, D., Keung, X.Z., Holm, T., Rings, A., DiAntonio, A., Hallermann, S., Ashery, U., et al. (2014). Quantitative super-resolution imaging of Bruchpilot distinguishes active zone states. Nat Commun 5, 4650.

Eriksen, J., Jorgensen, T.N., and Gether, U. (2010). Regulation of dopamine transporter function by protein-protein interactions: new discoveries and methodological challenges. J Neurochem.

Ester, M., Kriegel, H.-P., Sander, J., and Xu, X. (1996). A density-based algorithm for discovering clusters in large spatial databases with noise. In Proc 2nd International Conference on Knowledge Discovery and Data Mining (AAAI Press), pp. 226-231.

German, C.L., Baladi, M.G., McFadden, L.M., Hanson, G.R., and Fleckenstein, A.E. (2015). Regulation of the Dopamine and Vesicular Monoamine Transporters: Pharmacological Targets and Implications for Disease. Pharmacol Rev 67, 1005-1024.

Giguere, N., Delignat-Lavaud, B., Herborg, F., Voisin, A., Li, Y., Jacquemet, V., Anand-Srivastava, M., Gether, U., Giros, B., and Trudeau, L.E. (2019). Increased vulnerability of nigral dopamine neurons after expansion of their axonal arborization size through D2 dopamine receptor conditional knockout. PLoS Genet 15, e1008352.

Goncalves, J., Bartol, T.M., Camus, C., Levet, F., Menegolla, A.P., Sejnowski, T.J., Sibarita, J.B., Vivaudou, M., Choquet, D., and Hosy, E. (2020). Nanoscale co-organization and coactivation of AMPAR, NMDAR, and mGluR at excitatory synapses. Proc Natl Acad Sci U S A 117, 14503-14511.

Groc, L., and Choquet, D. (2020). Linking glutamate receptor movements and synapse function. Science 368.

Guthrie, D.A., Klein Herenbrink, C., Lycas, M.D., Ku, T., Bonifazi, A., DeVree, B.T., Mathiasen, S., Javitch, J.A., Grimm, J.B., Lavis, L., et al. (2020). Novel Fluorescent Ligands Enable Single-Molecule Localization Microscopy of the Dopamine Transporter. ACS Chem Neurosci 11, 3288-3300.

Hamilton, P.J., Belovich, A.N., Khelashvili, G., Saunders, C., Erreger, K., Javitch, J.A., Sitte, H.H., Weinstein, H., Matthies, H.J., and Galli, A. (2014). PIP2 regulates psychostimulant behaviors through its interaction with a membrane protein. Nat Chem Biol 10, 582-589. 
Hansen, F.H., Skjorringe, T., Yasmeen, S., Arends, N.V., Sahai, M.A., Erreger, K., Andreassen, T.F., Holy, M., Hamilton, P.J., Neergheen, V., et al. (2014). Missense dopamine transporter mutations associate with adult parkinsonism and ADHD. J Clin Invest 124, 3107-3120.

Heilemann, M., van de Linde, S., Schuttpelz, M., Kasper, R., Seefeldt, B., Mukherjee, A., Tinnefeld, P., and Sauer, M. (2008). Subdiffraction-resolution fluorescence imaging with conventional fluorescent probes. Angew Chem Int Ed Engl 47, 6172-6176.

Herborg, F., Andreassen, T.F., Berlin, F., Loland, C.J., and Gether, U. (2018). Neuropsychiatric diseaseassociated genetic variants of the dopamine transporter display heterogeneous molecular phenotypes. J Biol Chem 293, 7250-7262.

Hong, W.C., and Amara, S.G. (2010). Membrane cholesterol modulates the outward facing conformation of the dopamine transporter and alters cocaine binding. J Biol Chem 285, 32616-32626.

Huang, B., Bates, M., and Zhuang, X. (2009). Super-resolution fluorescence microscopy. Annu Rev Biochem 78, 993-1016.

Hwang, J., Tamm, L.K., Bohm, C., Ramalingam, T.S., Betzig, E., and Edidin, M. (1995). Nanoscale complexity of phospholipid monolayers investigated by near-field scanning optical microscopy. Science 270, 610-614.

Iversen, L.L. (1975). Dopamine receptors in the brain. Science 188, 1084-1089.

Jeong, J.Y., Yim, H.S., Ryu, J.Y., Lee, H.S., Lee, J.H., Seen, D.S., and Kang, S.G. (2012). One-step sequenceand ligation-independent cloning as a rapid and versatile cloning method for functional genomics studies. Appl Environ Microbiol 78, 5440-5443.

Klewe, I.V., Nielsen, S.M., Tarpo, L., Urizar, E., Dipace, C., Javitch, J.A., Gether, U., Egebjerg, J., and Christensen, K.V. (2008). Recruitment of beta-arrestin2 to the dopamine D2 receptor: insights into antipsychotic and anti-parkinsonian drug receptor signaling. Neuropharmacology 54, 1215-1222.

Kristensen, A.S., Andersen, J., Jorgensen, T.N., Sorensen, L., Eriksen, J., Loland, C.J., Stromgaard, K., and Gether, U. (2011). SLC6 neurotransmitter transporters: structure, function, and regulation. Pharmacol Rev 63, 585-640.

Kuzhikandathil, E.V., Yu, W.F., and Oxford, G.S. (1998). Human dopamine D3 and D2L receptors couple to inward rectifier potassium channels in mammalian cell lines. Molecular and Cellular Neuroscience 12, 390-402.

Lee, F.J., Pei, L., Moszczynska, A., Vukusic, B., Fletcher, P.J., and Liu, F. (2007). Dopamine transporter cell surface localization facilitated by a direct interaction with the dopamine D2 receptor. Embo J 26, 21272136.

Levet, F., Julien, G., Galland, R., Butler, C., Beghin, A., Chazeau, A., Hoess, P., Ries, J., Giannone, G., and Sibarita, J.B. (2019). A tessellation-based colocalization analysis approach for single-molecule localization microscopy. Nat Commun 10, 2379.

Li, Y.M., Mund, M., Hoess, P., Deschamps, J., Matti, U., Nijmeijer, B., Sabinina, V.J., Ellenberg, J., Schoen, I., and Ries, J. (2018). Real-time 3D single-molecule localization using experimental point spread functions. Nat Methods 15, 367-+.

Lin, C.-W., Sim, S., Ainsworth, A., Okada, M., Kelsch, W., and Lois, C. (2010). Genetically increased cellintrinsic excitability enhances neuronal integration into adult brain circuits. Neuron 65, 32-39. 
Liu, C., and Kaeser, P.S. (2019). Mechanisms and regulation of dopamine release. Curr Opin Neurobiol 57, 46-53.

Loland, C.J., Desai, R.I., Zou, M.F., Cao, J., Grundt, P., Gerstbrein, K., Sitte, H.H., Newman, A.H., Katz, J.L., and Gether, U. (2008). Relationship between conformational changes in the dopamine transporter and cocaine-like subjective effects of uptake inhibitors. Mol Pharmacol 73, 813-823.

Loland, C.J., Granas, C., Javitch, J.A., and Gether, U. (2004). Identification of intracellular residues in the dopamine transporter critical for regulation of transporter conformation and cocaine binding. J Biol Chem 279, 3228-3238.

Maidorn, M., Olichon, A., Rizzoli, S.O., and Opazo, F. (2019). Nanobodies reveal an extra-synaptic population of SNAP-25 and Syntaxin 1A in hippocampal neurons. Mabs 11, 305-321.

Manley, S., Gillette, J.M., Patterson, G.H., Shroff, H., Hess, H.F., Betzig, E., and Lippincott-Schwartz, J. (2008). High-density mapping of single-molecule trajectories with photoactivated localization microscopy. Nat Methods 5, 155-157.

Maxfield, F.R., and McGraw, T.E. (2004). Endocytic recycling. Nature Reviews Molecular Cell Biology 5, 121-132.

McLaughlin, S., and Murray, D. (2005). Plasma membrane phosphoinositide organization by protein electrostatics. Nature 438.

Meiergerd, S.M., Patterson, T.A., and Schenk, J.O. (1993). D2 receptors may modulate the function of the striatal transporter for dopamine - kinetic evidence from studies in-vitro and in-vivo. Journal of Neurochemistry 61, 764-767.

Newman, A.H., Cao, J., Keighron, J.D., Jordan, C.J., Bi, G.H., Liang, Y., Abramyan, A.M., Avelar, A.J., Tschumi, C.W., Beckstead, M.J., et al. (2019). Translating the atypical dopamine uptake inhibitor hypothesis toward therapeutics for treatment of psychostimulant use disorders.

Neuropsychopharmacology 44, 1435-1444.

Ovesny, M., Krizek, P., Borkovec, J., Svindrych, Z., and Hagen, G.M. (2014). ThunderSTORM: a comprehensive ImageJ plug-in for PALM and STORM data analysis and super-resolution imaging. Bioinformatics 30, 2389-2390.

Padmanabhan, P., Bademosi, A.T., Kasula, R., Lauwers, E., Verstreken, P., and Meunier, F.A. (2020). Need for speed: Super-resolving the dynamic nanoclustering of syntaxin-1 at exocytic fusion sites. Neuropharmacology 169.

Parsons, L.H., Schad, C.A., and Justice, J.B. (1993). Coadministration of the $\mathrm{d} 2$ antagonist pimozide inhibits up-regulation of dopamine release and uptake induced by repeated cocaine. Journal of Neurochemistry 60, 376-379.

Pereira, D.B., Schmitz, Y., Meszaros, J., Merchant, P., Hu, G., Li, S., Henke, A., Lizardi-Ortiz, J.E., Karpowicz, R.J., Jr., Morgenstern, T.J., et al. (2016). Fluorescent false neurotransmitter reveals functionally silent dopamine vesicle clusters in the striatum. Nat Neurosci 19, 578-586.

Qi, Y., Wang, J.K., McMillian, M., and Chikaraishi, D.M. (1997). Characterization of a CNS cell line, CAD, in which morphological differentiation is initiated by serum deprivation. J Neurosci 17, 1217-1225.

Rahbek-Clemmensen, T., Lycas, M.D., Erlendsson, S., Eriksen, J., Apuschkin, M., Vilhardt, F., Jorgensen, T.N., Hansen, F.H., and Gether, U. (2017). Super-resolution microscopy reveals functional organization 
of dopamine transporters into cholesterol and neuronal activity-dependent nanodomains. Nat Commun 8,740 .

Ramamoorthy, S., Shippenberg, T.S., and Jayanthi, L.D. (2011). Regulation of monoamine transporters: Role of transporter phosphorylation. Pharmacol Ther 129, 220-238.

Rayport, S., Sulzer, D., Shi, W.X., Sawasdikosol, S., Monaco, J., Batson, D., and Rajendran, G. (1992). Identified postnatal mesolimbic dopamine neurons in culture: morphology and electrophysiology. J Neurosci 12, 4264-4280.

Robertson, S.D., Matthies, H.J., and Galli, A. (2009). A closer look at amphetamine-induced reverse transport and trafficking of the dopamine and norepinephrine transporters. Mol Neurobiol 39, 73-80.

Salamone, A., Zappettini, S., Grilli, M., Olivero, G., Agostinho, P., Tome, A.R., Chen, J., Pittaluga, A., Cunha, R.A., and Marchi, M. (2014). Prolonged nicotine exposure down-regulates presynaptic NMDA receptors in dopaminergic terminals of the rat nucleus accumbens. Neuropharmacology 79, 488-497.

Shivanandan, A., Deschout, H., Scarselli, M., and Radenovic, A. (2014). Challenges in quantitative single molecule localization microscopy. FEBS Lett 588, 3595-3602.

Siddig, S., Aufmkolk, S., Doose, S., Jobin, M.-L., Werner, C., Sauer, M., and Calebiro, D. (2020). Superresolution imaging reveals the nanoscale organization of metabotropic glutamate receptors at presynaptic active zones. Science Advances 6, eaay7193.

Strange, P.G. (2008). Antipsychotic drug action: antagonism, inverse agonism or partial agonism. Trends Pharmacol Sci 29, 314-321.

Suh, B.C., and Hille, B. (2008). PIP2 is a necessary cofactor for ion channel function: how and why? Annu Rev Biophys 37, 175-195.

Tillberg, P.W., Chen, F., Piatkevich, K.D., Zhao, Y., Yu, C.C., English, B.P., Gao, L., Martorell, A., Suk, H.J., Yoshida, F., et al. (2016). Protein-retention expansion microscopy of cells and tissues labeled using standard fluorescent proteins and antibodies. Nat Biotechnol 34, 987-992.

Torres, G.E. (2006). The dopamine transporter proteome. J Neurochem 97 Suppl 1, 3-10.

Torres, G.E., and Amara, S.G. (2007). Glutamate and monoamine transporters: new visions of form and function. Curr Opin Neurobiol 17, 304-312.

Tritsch, N.X., and Sabatini, B.L. (2012). Dopaminergic modulation of synaptic transmission in cortex and striatum. Neuron 76, 33-50.

Truckenbrodt, S., Sommer, C., Rizzoli, S.O., and Danzl, J.G. (2019). A practical guide to optimization in X10 expansion microscopy. Nat Protoc 14, 832-863.

Turkowyd, B., Schreiber, S., Wortz, J., Segal, E.S., Mevarech, M., Duggin, I.G., Marchfelder, A., and Endesfelder, U. (2020). Establishing Live-Cell Single-Molecule Localization Microscopy Imaging and Single-Particle Tracking in the Archaeon Haloferax volcanii. Front Microbiol 11, 583010.

Turner, T.J., Adams, M.E., and Dunlap, K. (1993). Multiple Ca2+ channel types coexist to regulate synaptosomal neurotransmitter release. Proceedings of the National Academy of Sciences of the United States of America 90, 9518-9522.

Underhill, S.M., Wheeler, D.S., Li, M., Watts, S.D., Ingram, S.L., and Amara, S.G. (2014). Amphetamine modulates excitatory neurotransmission through endocytosis of the glutamate transporter EAAT3 in dopamine neurons. Neuron 83, 404-416. 
van den Bogaart, G., Meyenberg, K., Risselada, H.J., Amin, H., Willig, K.I., Hubrich, B.E., Dier, M., Hell, S.W., Grubmuller, H., Diederichsen, U., et al. (2011). Membrane protein sequestering by ionic proteinlipid interactions. Nature 479, 552-555.

Wang, Y., Schnitzbauer, J., Hu, Z., Li, X., Cheng, Y., Huang, Z.L., and Huang, B. (2014). Localization eventsbased sample drift correction for localization microscopy with redundant cross-correlation algorithm. Opt Express 22, 15982-15991.

Xue, M., Atallah, B.V., and Scanziani, M. (2014). Equalizing excitation-inhibition ratios across visual cortical neurons. Nature 511, 596-600.

Zapata, A., Kivell, B., Han, Y., Javitch, J.A., Bolan, E.A., Kuraguntla, D., Jaligam, V., Oz, M., Jayanthi, L.D., Samuvel, D.J., et al. (2007). Regulation of dopamine transporter function and cell surface expression by D3 dopamine receptors. J Biol Chem 282, 35842-35854. 
Fig. 1

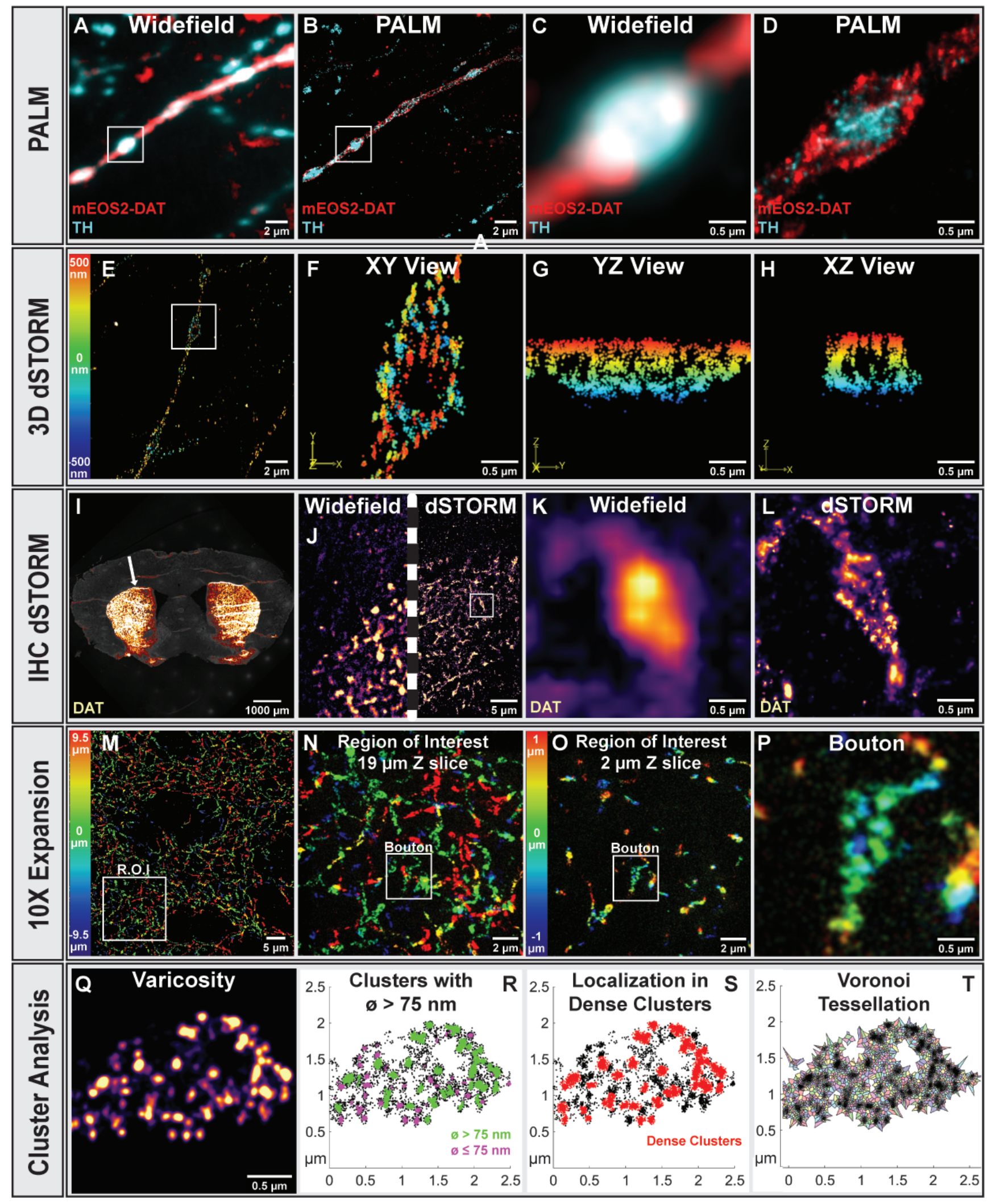


Fig. 2
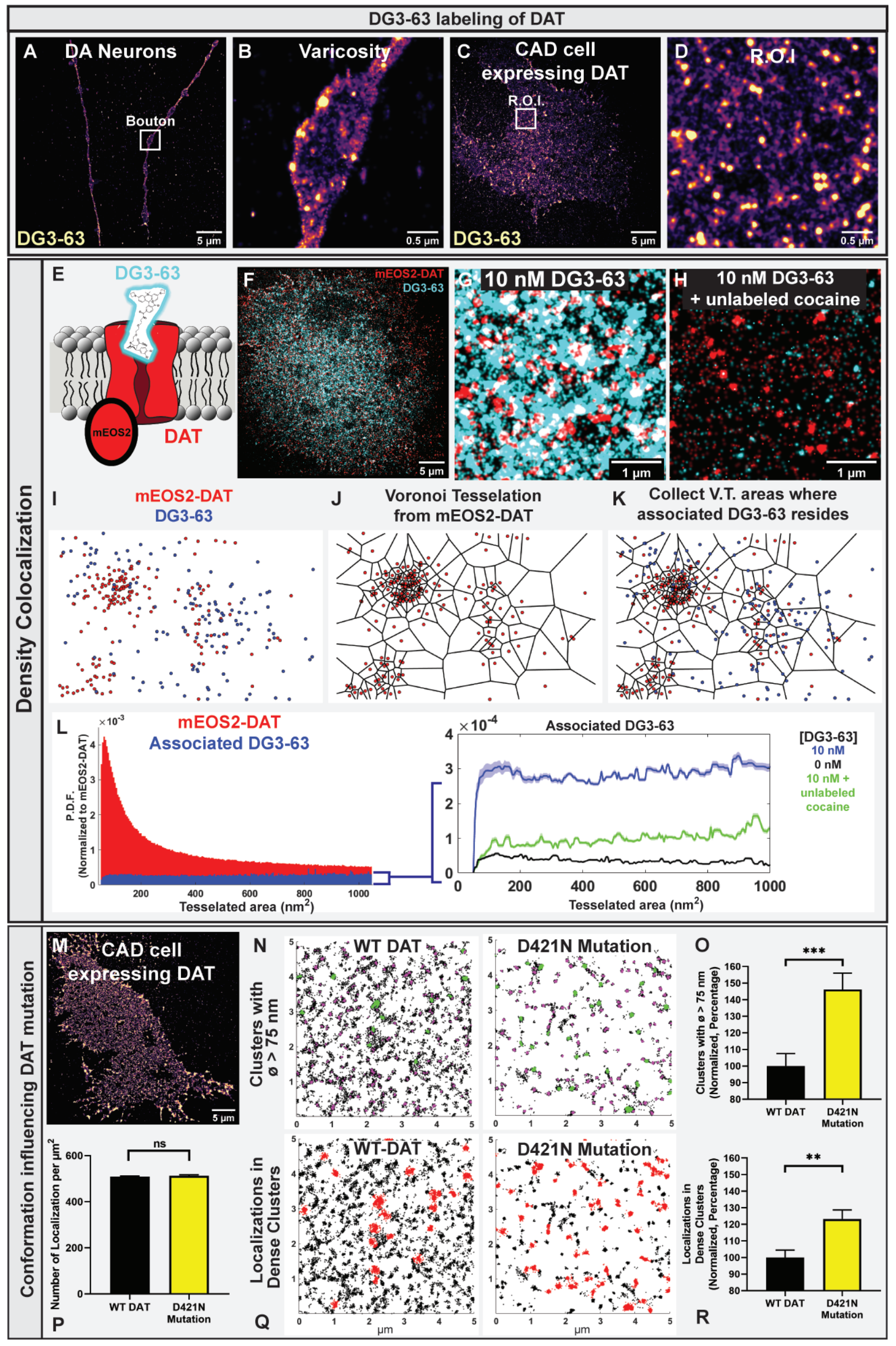
bioRxiv preprint doi: https://doi.org/10.1101/2021.03.09.434538; this version posted March 10, 2021. The copyright holder for this preprint

Fig. 3
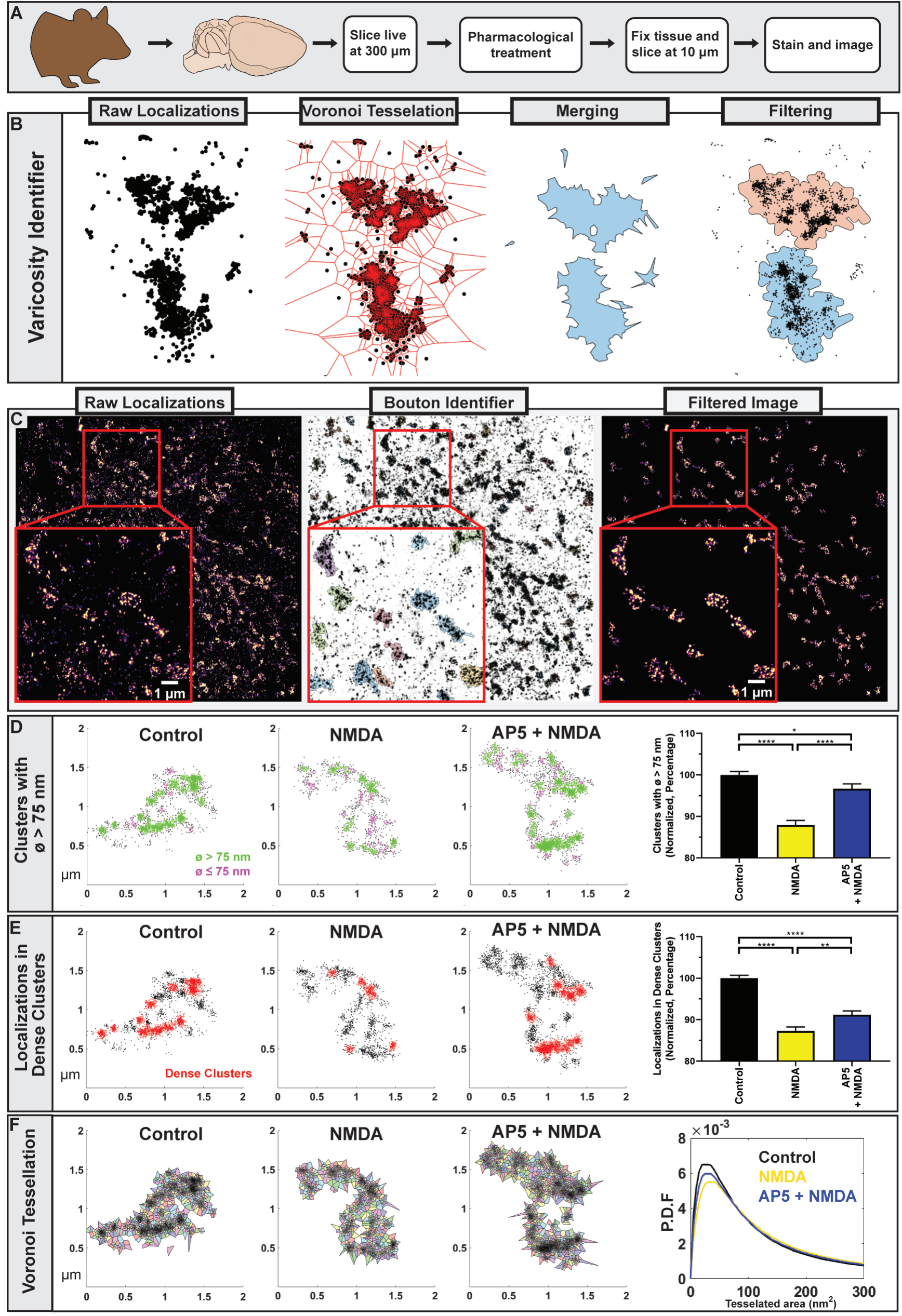
bioRxiv preprint doi: https://doi.org/10.1101/2021.03.09.434538; this version posted March 10, 2021. The copyright holder for this preprint

Fig. 4

(which was not certified by peer review) is the author/funder. All rights reserved. No reuse allowed without permission.
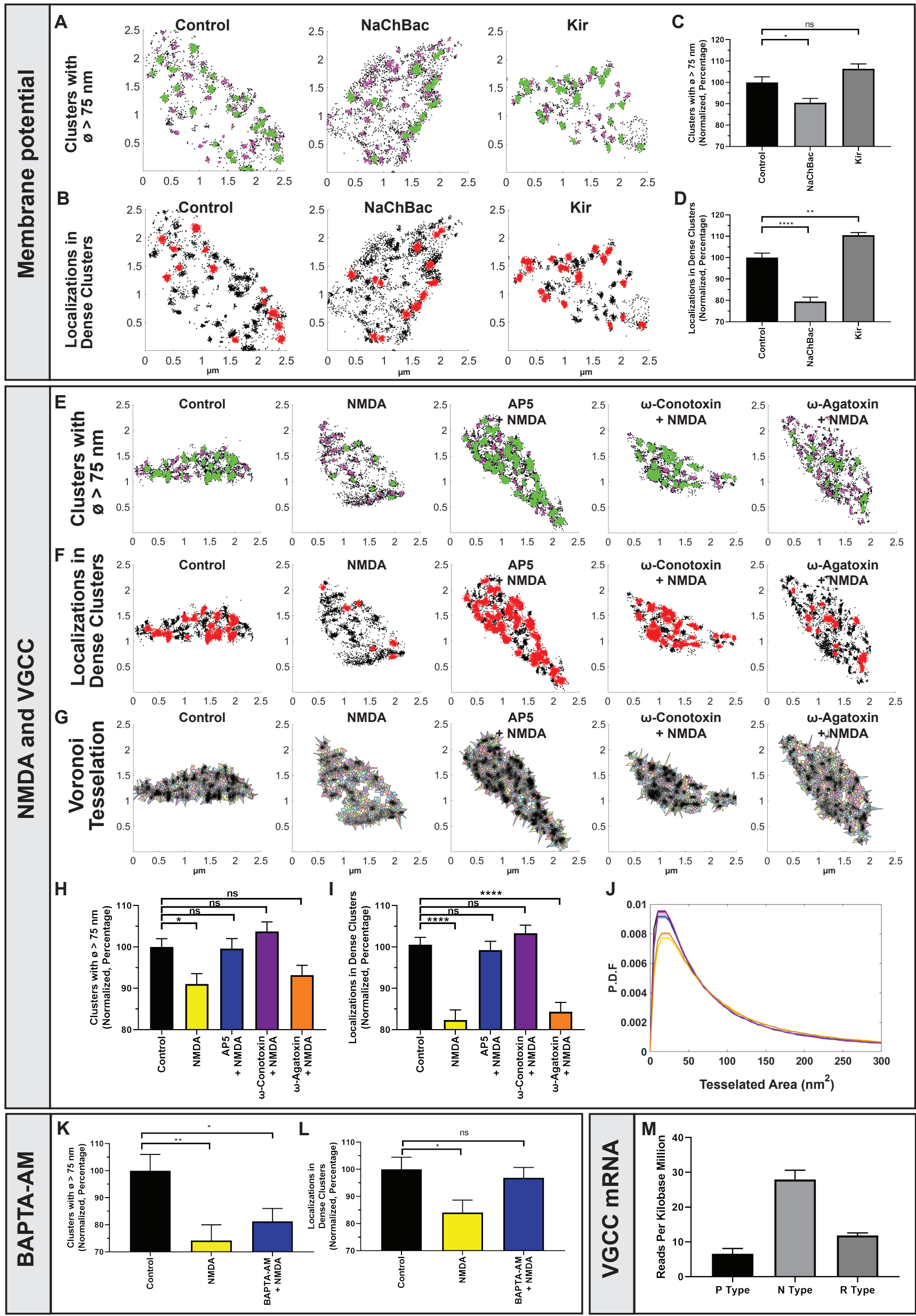
bioRxiv preprint doi: https://doi.org/10.1101/2021.03.09.434538; this version posted March 10, 2021. The copyright holder for this preprint (which was not certified by peer review) is the author/funder. All rights reserved. No reuse allowed without permission.

Fig. 5
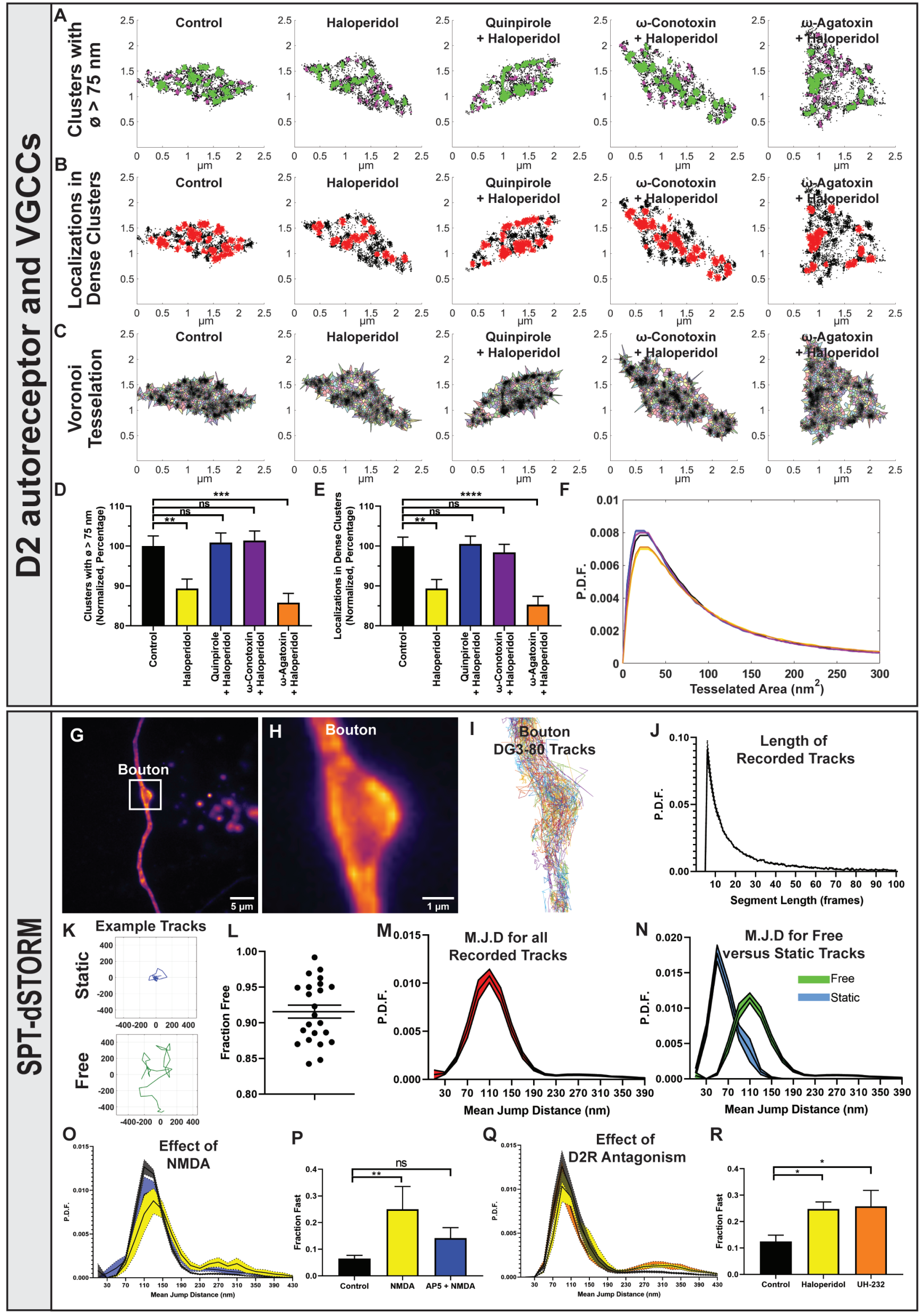
Fig. 6

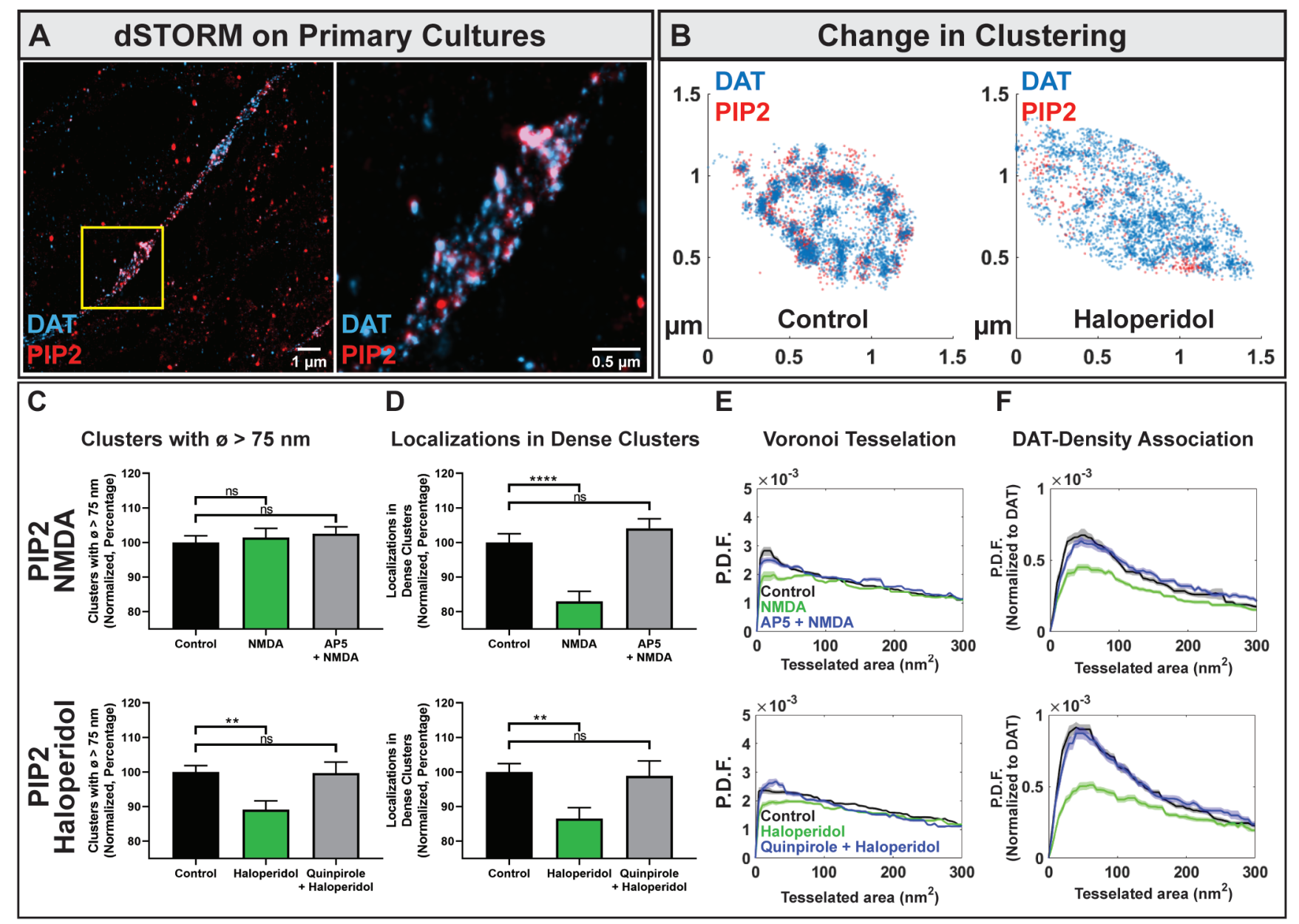




\section{Fig. 7}

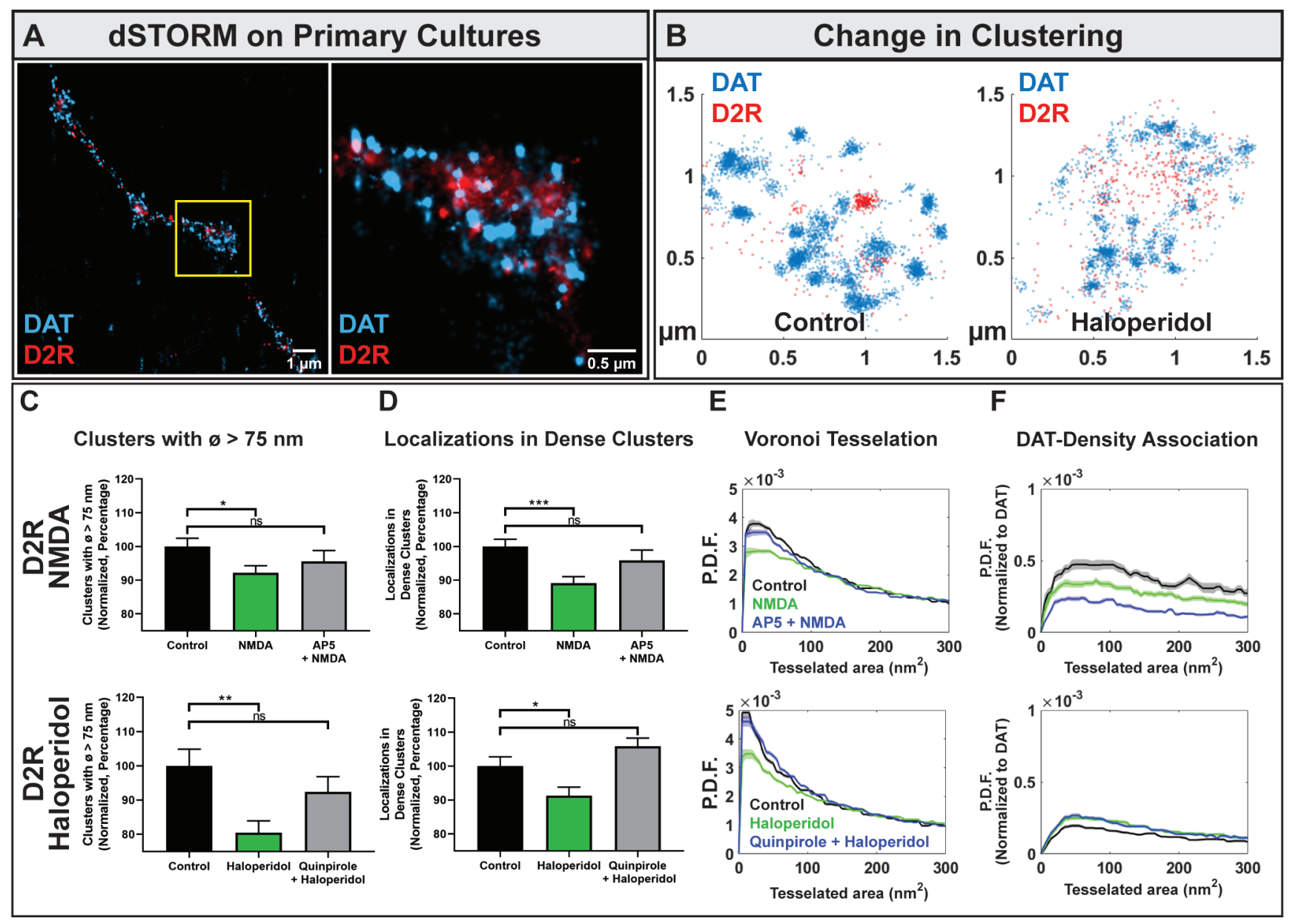


bioRxiv preprint doi: https://doi.org/10.1101/2021.03.09.434538; this version posted March 10, 2021. The copyright holder for this preprint (which was not certified by peer review) is the author/funder. All rights reserved. No reuse allowed without permission.

\section{Fig. S1}
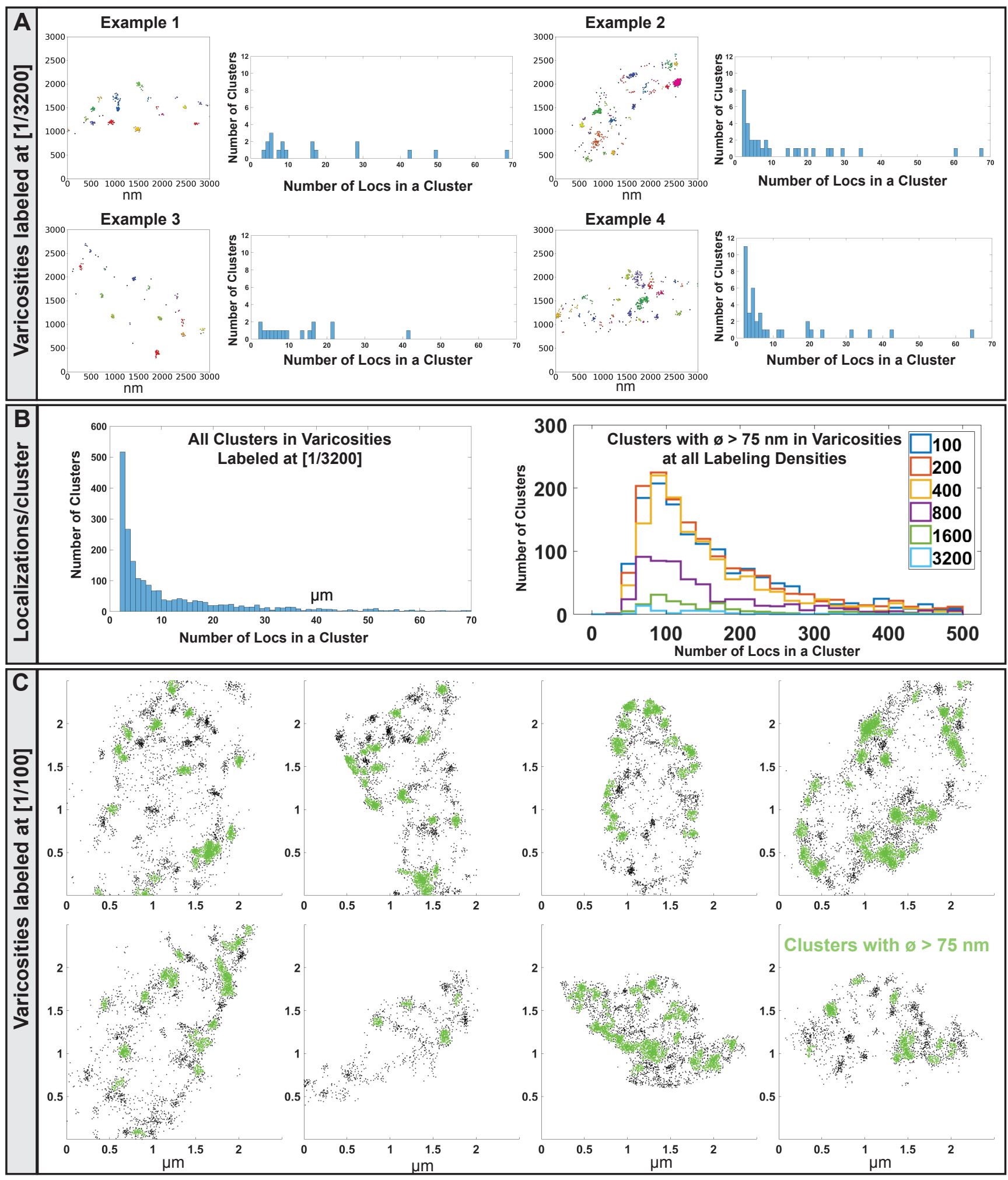

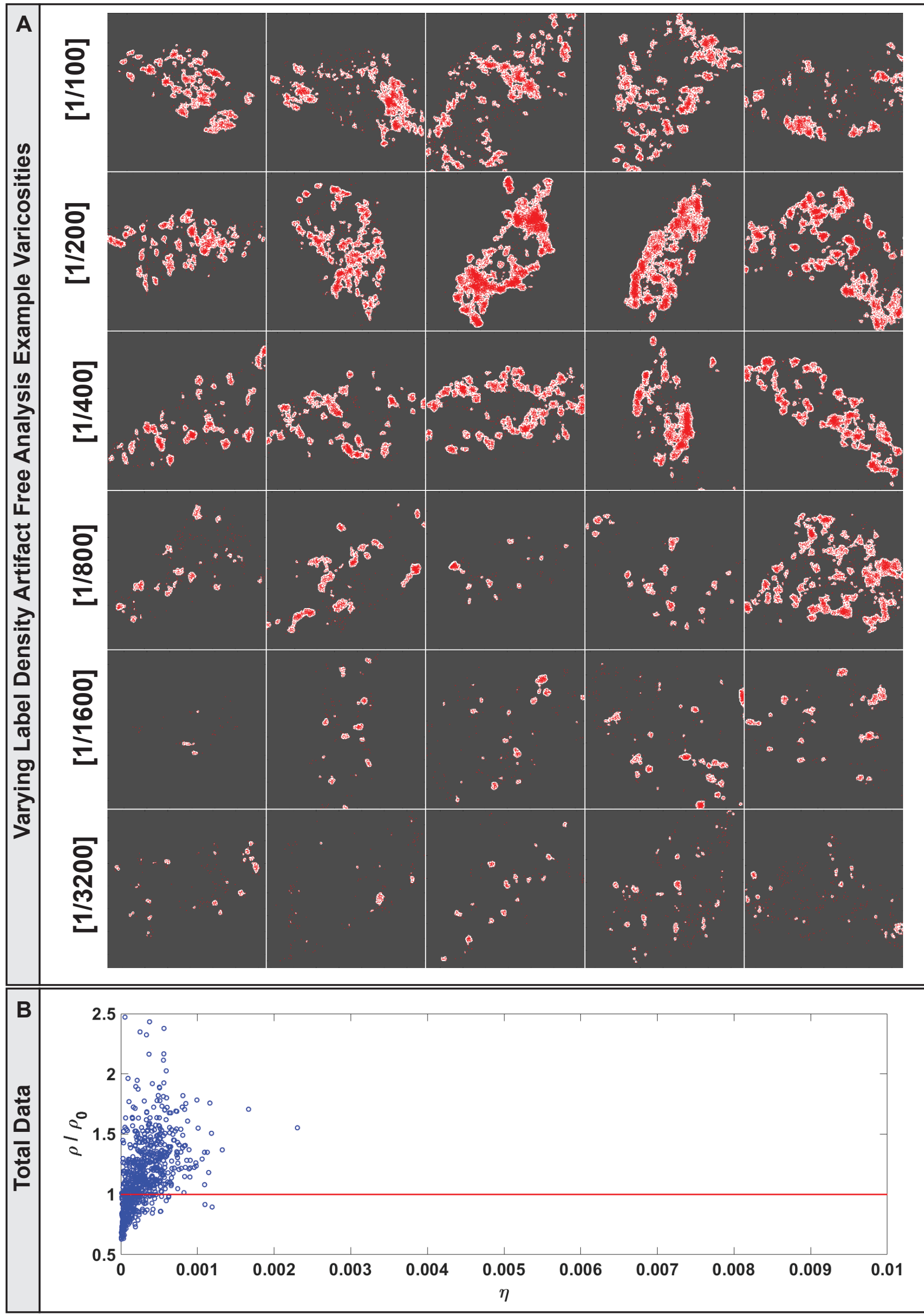


\section{Fig. S3}

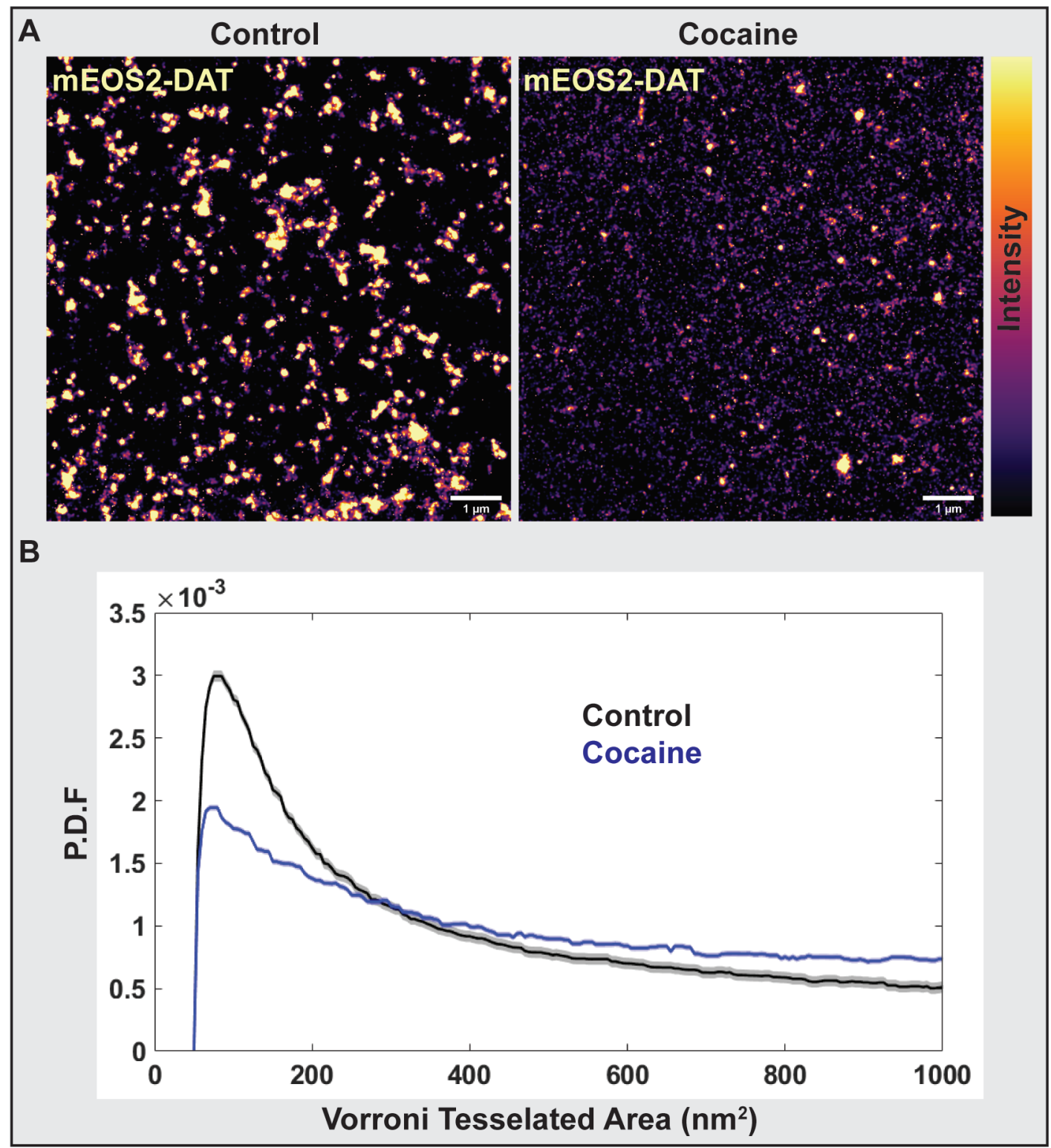




\section{Fig. S4}

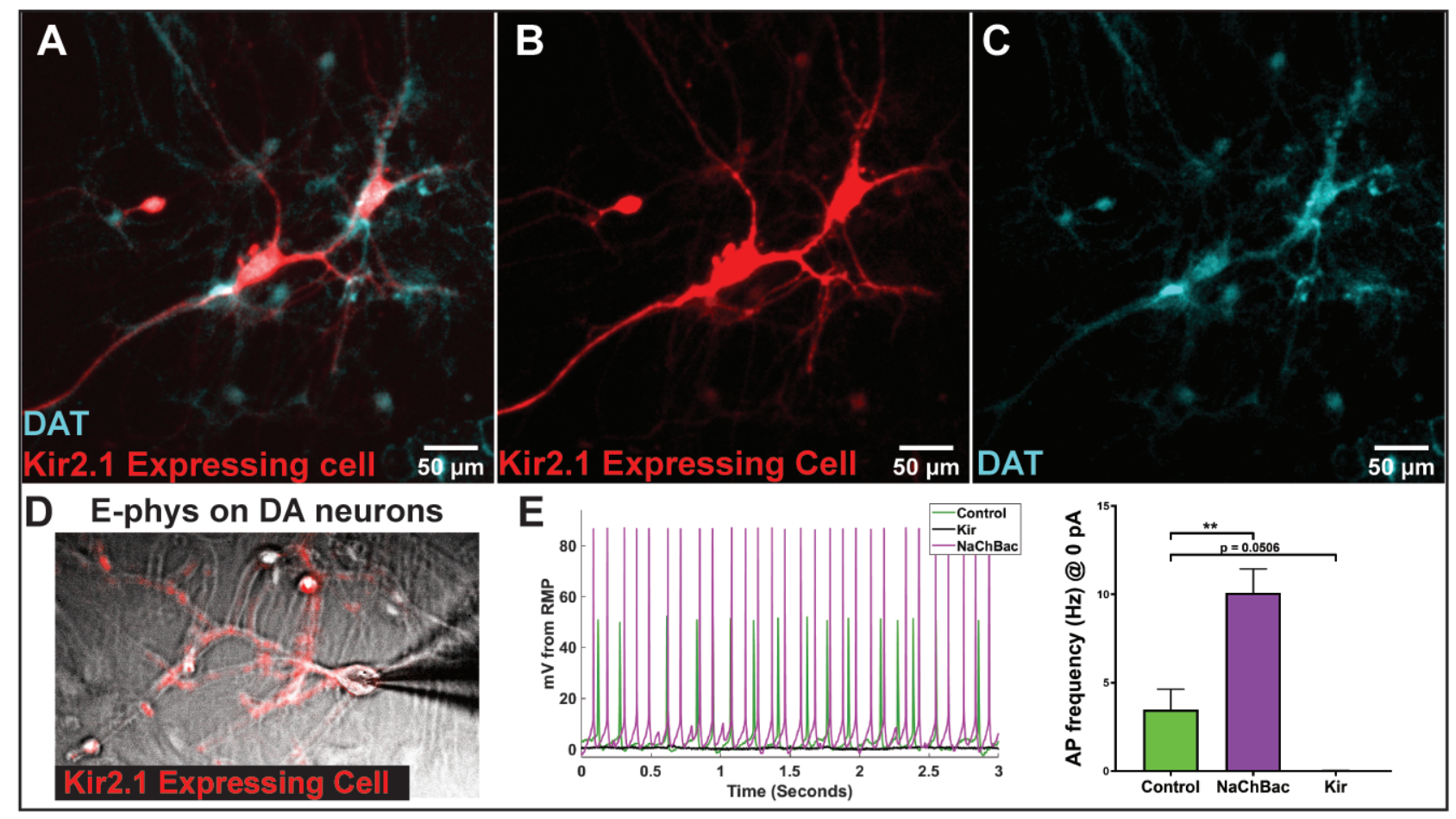


Fig. S5

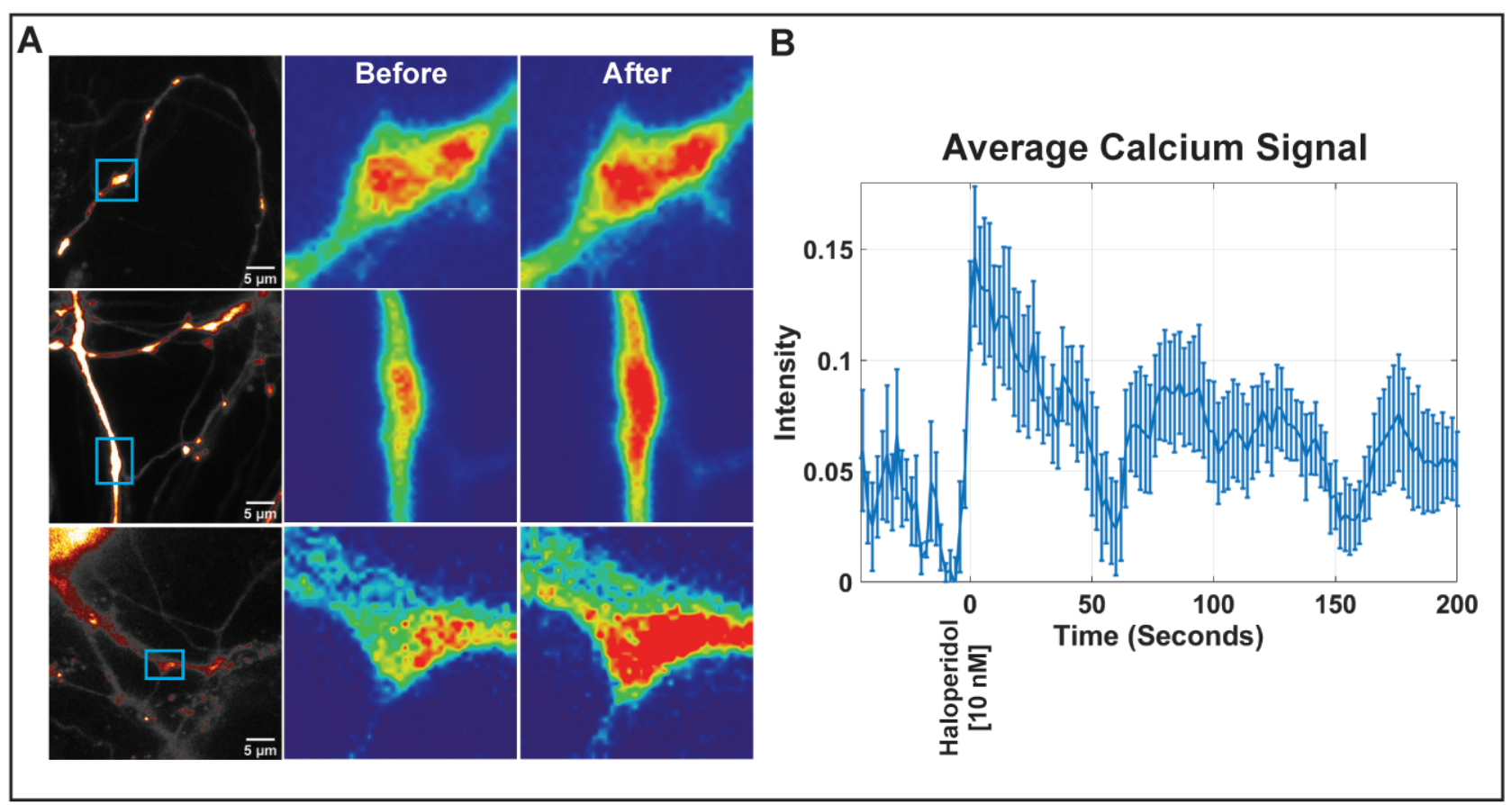

\section{C}
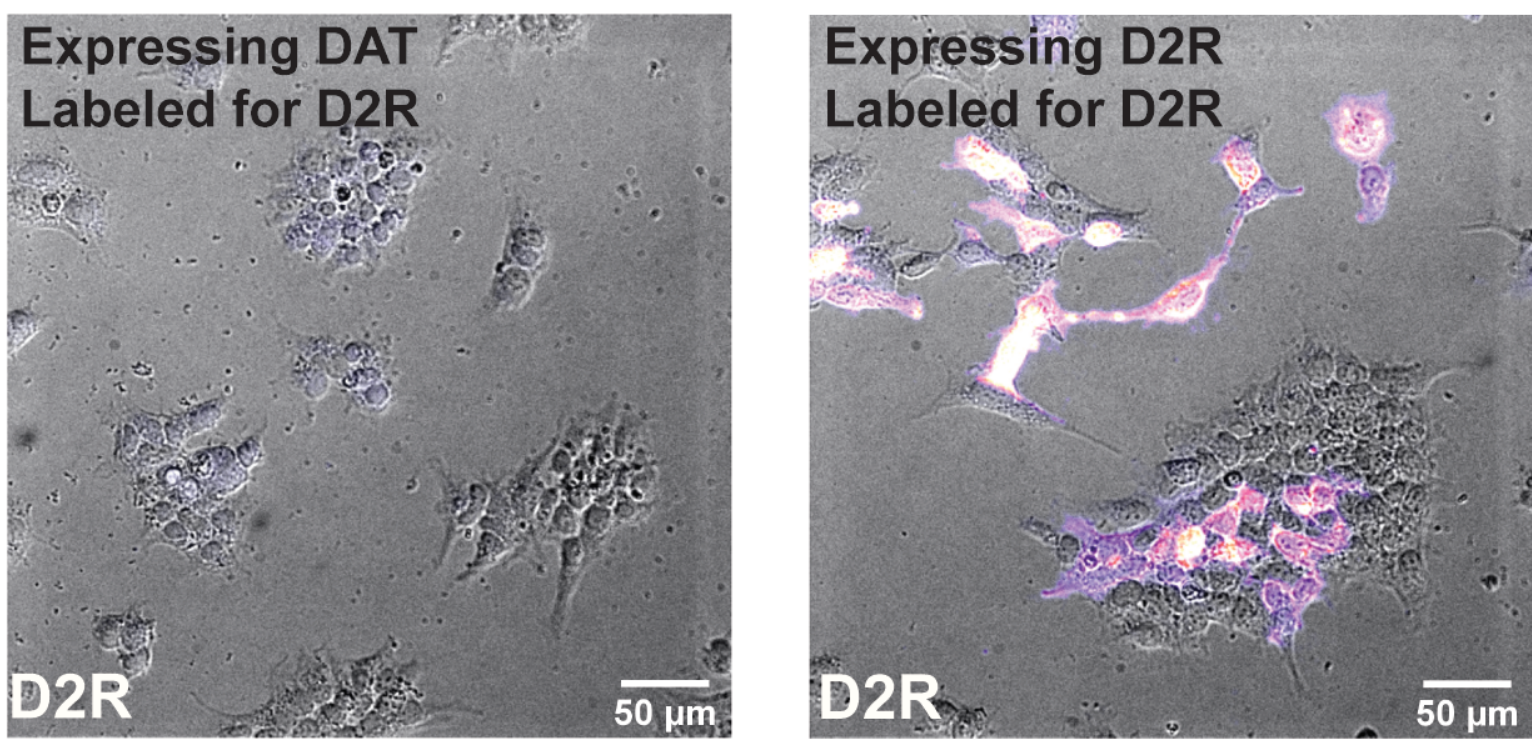


\section{Fig. $\mathbf{6} 6$}
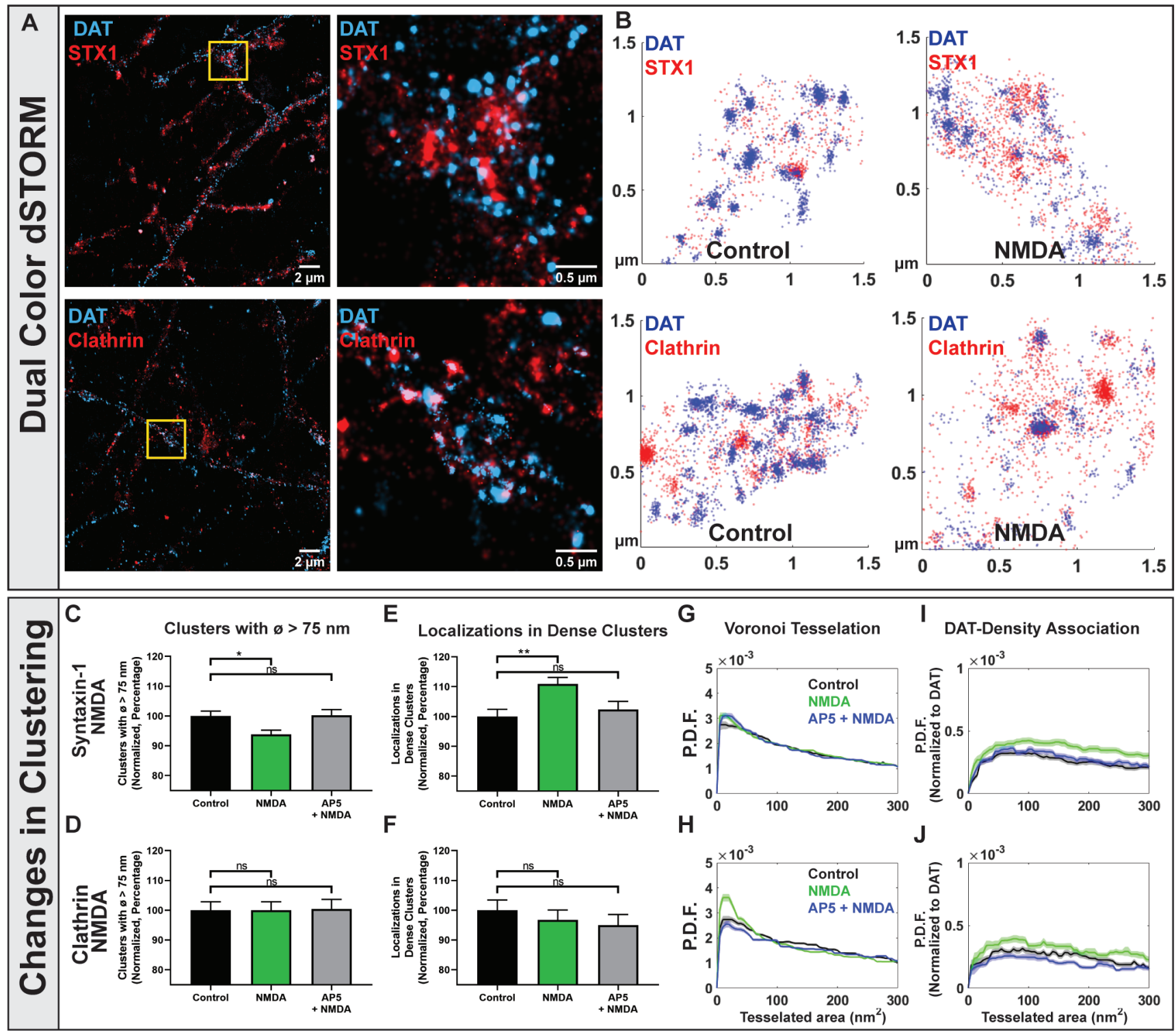
bioRxiv preprint doi: https://doi.org/10.1101/2021.03.09.434538; this version posted March 10, 2021. The copyright holder for this preprint Fig. 57

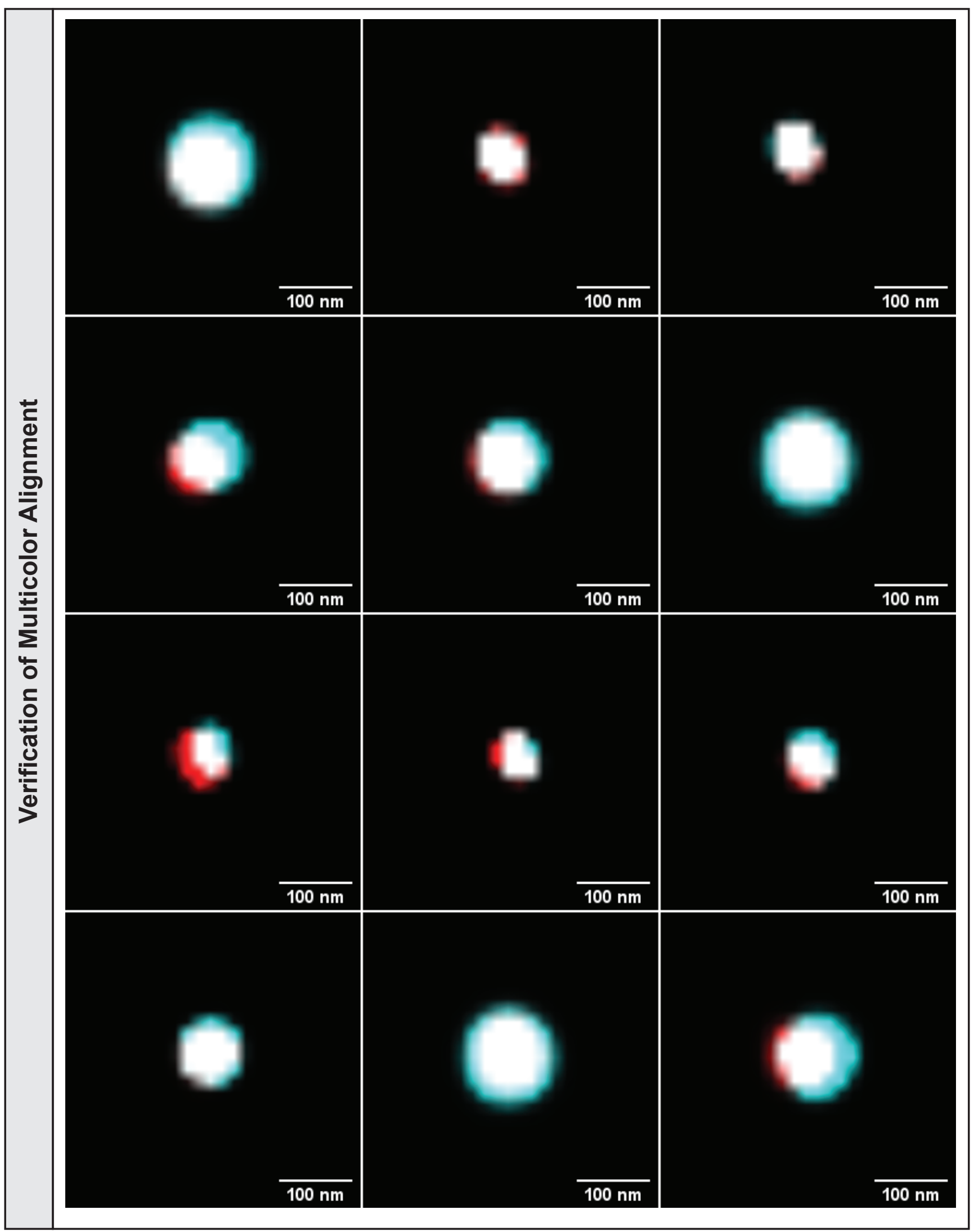

